\title{
Gravitational waves from extreme mass ratio inspirals in nonpure Kerr spacetimes
}

\author{
Enrico Barausse, ${ }^{1}$ Luciano Rezzolla, ${ }^{2,3}$ David Petroff, ${ }^{4}$ and Marcus Ansorg ${ }^{2}$ \\ ${ }^{1}$ SISSA, International School for Advanced Studies and INFN, Trieste, Italy \\ ${ }^{2}$ Max-Planck-Institut für Gravitationsphysik, Albert-Einstein-Institut, Potsdam-Golm, Germany \\ ${ }^{3}$ Department of Physics, Louisiana State University, Baton Rouge, USA \\ ${ }^{4}$ Theoretisch-Physikalisches Institut, University of Jena, Jena, Germany
}

(Received 20 December 2006; published 21 March 2007)

To investigate the imprint on the gravitational-wave emission from extreme mass ratio inspirals (EMRIs) in nonpure Kerr spacetimes, we have studied the kludge waveforms generated in highly accurate, numerically generated spacetimes containing a black hole and a self-gravitating, homogeneous torus with comparable mass and spin. In order to maximize their impact on the produced waveforms, we have considered tori that are compact, massive, and close to the central black hole, investigating under what conditions the LISA experiment could detect their presence. Our results show that for a large portion of the space of parameters the waveforms produced by EMRIs in these black hole-torus systems are indistinguishable from pure Kerr waveforms. Hence, a "confusion problem" will be present for observations carried out over a time scale below or comparable to the dephasing time.

DOI: 10.1103/PhysRevD.75.064026

PACS numbers: 04.30. $-\mathrm{w}, 04.70 .-\mathrm{s}, 98.35 . J k, 98.62 . J \mathrm{~s}$

\section{INTRODUCTION}

Extreme mass ratio inspirals (EMRIs) are thought to be one of the most interesting sources of gravitational waves for the space-based gravitational-wave detector LISA [1]: the typical example is a black hole with mass $\sim 1-10 M_{\odot}$ orbiting around the supermassive black hole (SMBH) at the center of a galaxy. It is expected that LISA will be able to detect anywhere from tens up to a thousand of these sources during its lifetime, which will probably be between 3 and 5 years. Although the masses of SMBHs range from $10^{6}$ to $10^{10} M_{\odot}$ [2], the mass of the SMBH involved in an EMRI must be around $10^{6} M_{\odot}$ in order for the gravitationalwave signal to be within LISA's sensitivity band: see, for instance, Ref. [3] for the expected event rates for different masses of the stellar black hole and of the SMBH.

As in the case of Earth-based detectors, for which the signal is generally expected to be comparable with the noise, the detection of gravitational waves emitted by EMRIs and the subsequent characterization of the source is expected to take place at small values of the signalto-noise ratio (SNR), thus requiring some sort of matched filtering. This method is based on cross correlating the noisy gravitational-wave signal with a bank of templates, which should accurately model the true signal, and poses serious challenges both in building the templates and in accessing them (see Ref. [3] for a detailed discussion).

The SMBHs involved in EMRIs are commonly thought to be describable by the pure Kerr solution of general relativity: this is the common assumption made in most work on EMRIs. Nevertheless, a number of other "exotic" candidates have been proposed as alternatives to the central massive object. These are, for instance, gravastars [4], boson stars [5], fermion balls [6], oscillating axion bubbles [7], etc. Clearly, while it is not yet possible to exclude completely these possibilities, the presence of these objects at the centers of galaxies would require a serious modification to the scenarios through which galaxies are expected to form. At the same time, the possibility that LISA observations could be used to determine the presence (or absence) of these objects, provides additional scientific value to this challenging experiment.

Hereafter, we will adopt a more conservative view and assume that the central object is indeed an SMBH. Recent observations of the near-infrared fluxes of SgrA* support this view by setting upper limits on the mass accretion rate of the galactic center and showing that the central massive object must have, under reasonable assumptions, an event horizon [8]. Yet, even with this assumption, the modelling of EMRIs can in principle suffer from the uncertainty of whether the spacetime in the vicinity of the SMBH can be accurately described in terms of a (pure) Kerr solution. The origin of this uncertainty is that SMBHs are not expected to be in vacuum and indeed a considerable amount of matter is expected to be present around the central massive object. In the case of active galactic nuclei (AGNs), for instance, the intense high-energy emission is thought to be the result of a pc-scale accretion disk (and perhaps a thick torus) extending down almost to the innermost stable circular orbit (ISCO), feeding the central black hole. In addition, a dusty obscuration torus is also believed to be present on much larger scales (i.e., $\sim 10-100 \mathrm{pc}$ ) [9]. Too little is presently known about the properties of these disks and although their mass is commonly thought to be much smaller than the mass of the SMBH, there are observations hinting at disks as massive as the central object [10]. 
Another example is given by SgrA* itself, where counterrotating stellar disks on scales less than $1 \mathrm{pc}$ have been observed [11]. This is hardly surprising since the galactic center is expected to be a high density environment, as the distribution of stars shows a cusp there: the mass density in stars is believed to be $\rho \sim \rho_{0}\left(r / r_{0}\right)^{-\alpha}$, with $\rho_{0} \sim 1.2 \times$ $10^{6} M_{\odot} / \mathrm{pc}^{3}, r_{0} \sim 0.04 \mathrm{pc}$, and $\alpha \sim 1.4-2$ [11].

Furthermore, even if an SMBH exists, it is still possible that it could be surrounded by other, nonvisible components, such as clusters of compact objects or high concentrations of exotic particles. Cosmological $N$-body simulations predict, in fact, that the cold dark matter (CDM) density in galactic halos should show a "cusp" near the galactic center with a profile of the type [12] $\rho_{\mathrm{CDM}} \sim \rho_{0}\left(r / r_{0}\right)^{-\alpha}$, where $\alpha \sim 1$ : although the mass in CDM particles is generally thought to be smaller than that in the stellar cusp $\left(\rho_{0} \sim 100 M_{\odot} / \mathrm{pc}^{2}\right.$ and $r_{0} \sim 3 \mathrm{pc}$ [13]), the normalization of this power law is still very uncertain. ${ }^{1}$ In addition, although this CDM distribution is commonly thought to be spherically symmetric, the confrontation with observations still leaves a number of uncertainties, with the presence of the CDM cusp itself being in contrast with observations of galactic rotation curves, which instead hint at a CDM core-profile in galactic centers [16]. The possibility that CDM could be distributed along caustic rings in galactic halos has also been suggested [17].

Clearly, gravitational-wave observations through the LISA detector could shed some light on these issues, enabling the distinction between competing models for the central massive object and for the distribution of matter around it. Indeed, observations of EMRIs by LISA could allow us to build a map of the spacetime around galactic centers and determine with great precision the properties of the spacetime in regions which are not easily accessible through electromagnetic observations.

A number of different approaches to this "spacetimemapping" problem were considered in the literature: EMRIs have been studied in spacetimes which are either approximate or exact solutions of the Einstein equations. Among the former, a multipolar expansion suitable to describe general stationary, axisymmetric, asymptotically flat spacetimes outside a central distribution of matter has been considered [18-20]. However, this multipolar expansion is in practice a series in $1 / r$ ( $r$ being the distance to the central object) around a Minkowski spacetime: an accurate representation of the strong-field regime would require the inclusion of many terms. Another possibility is the "quasiKerr" (i.e., Kerr plus a small quadrupole) spacetime studied by Glampedakis and Babak [21]. This can approximately describe the spacetime outside a slowly rotating boson star and is not an expansion around Minkowski, thus

\footnotetext{
${ }^{1}$ The possibility that a steeper profile (i.e., larger $\alpha$ ) could form under the influence of the SMBH was also proposed in Ref. [14], although the process does not seem to happen in a more realistic astrophysical scenario [15].
}

being more promising in the strong-field limit. Among exact solutions of the Einstein equations, only spherical boson stars [22] and "bumpy black holes" [23] (i.e., objects that, although involving naked singularities, are almost Schwarzschild black holes, but have some multipoles with the wrong values) have been considered.

At any rate, none of these spacetimes, neither exact nor approximate, can describe satisfactorily the "astrophysical bumpiness" which is certainly present around SMBHs. With this in mind, we have studied EMRIs in stationary, axisymmetric spacetimes which are highly accurate numerical solutions of the Einstein equations and contain a rotating black hole and a torus [24].

We used these numerical spacetimes to perform a study similar to that carried out by Babak and Glampedakis for "quasi-Kerr" spacetimes [21]: we studied EMRIs in the equatorial plane and computed semirelativistic ("kludge") waveforms, comparing them to kludge, pure Kerr waveforms. Babak and Glampedakis, in particular, find there could be a "confusion" problem, because although gravitational waves emitted in a quasi-Kerr spacetime by a stellar mass black hole moving on an equatorial orbit are wildly different from those emitted by the same stellar mass black hole moving along the same orbit in a pure Kerr spacetime (having the same mass and spin as the quasi-Kerr spacetime), waveforms produced by equatorial orbits having slightly different latus rectum and eccentricity but the same $r$ - and $\phi$-frequencies turn out to be indistinguishable with LISA's sensitivity. We therefore repeated and extended their analysis. In particular, we introduce, like them, a suitable cutoff in time in order not to have any relevant radiation-reaction effects on the geodetic motion. While this could be avoided in Babak's and Glampedakis' quasi-Kerr spacetimes (probably eliminating the confusion problem: see the analysis in Ref. [25]), this is actually a necessity in our case, since the effect of a torus on the loss of energy and angular momentum due to gravitational-wave emission is completely unknown at the present.

We did not try, for the moment, to produce tori describing the accretion disk of AGNs (although we plan to do this in a future paper), but rather adopted a more phenomenological approach. Indeed, since little is known about the strong-field region near the central massive black hole, we tried to build some "extreme" configurations, i.e. configurations containing rather massive and compact tori (close to the event horizon of the central black hole). The purpose is to understand if LISA can detect the presence of such tori, which are so close to the horizon that they could not probably be detected otherwise (for instance, by means of stellar orbits), especially if made of some "dark" mass. We stress that the word extreme does not mean that these configurations are extremely far from Kerr, but just that these tori are not the ones astrophysicists expect in AGNs. 
One possible objection is that it might be possible that these extreme configurations are unstable (tackling the problem of stability is indeed one of the points in which the results of Ref. [24] may be improved in the future), but we do not think this should be a major concern at this stage. Our viewpoint is that considering such extreme configurations will provide a test bed to investigate the practical problems of spacetime-mapping through EMRIgravitational waves. In particular, these configurations will also help to understand better the confusion problem pointed out by Glampedakis and Babak. As already stressed, while in quasi-Kerr spacetimes this confusion disappears when dropping the time cutoff and including radiation reaction [25], in our case it may still be present due to the practical difficulties of computing radiation reaction in our spacetimes, which force us to introduce a cutoff in time.

We will see, however, that this confusion in the orbital parameters appears in our spacetimes only for (equatorial) orbits far from the black hole-torus system, whereas it disappears in the strong-field region. Nevertheless, we find another confusion problem, potentially more worrisome as it involves the parameters of the black hole. Of course, if we could replace the semirelativistic approximation with a rigorous solution of the linearized Einstein equations and a proper treatment of self-force or radiation reaction, this confusion problem may disappear as well. However, such a rigorous treatment is very hard to obtain in generic stationary and axisymmetric spacetimes (see Sec. II) and, as far as the self-force is considered, even in pure Kerr.

This paper is organized as follows. In Sec. II we show what the rigorous treatment of EMRIs in nonvacuum, stationary, and axisymmetric spacetimes would be, and explain why this treatment has proved so hard that nobody has pursued it so far. In Sec. III we review the non-Kerr spacetimes in which the problem of EMRIs has been considered to date, ranging from approximate (Sec. III A) to exact (Sec. III B) solutions of the Einstein equations, and we introduce the nonpure Kerr spacetimes we will use instead (Sec. III C). In Sec. IV we review the semirelativistic formalism used in Ref. [21] to compute gravitational waves and explain how we adapted it to our purposes; in particular, we show how we integrated the geodesic equations and calculated kludge waveforms, and (in Sec. IVA) explain what the overlap function and the dephasing time are. In Sec. V we explain in detail how we perform a comparison between our nonpure Kerr spacetimes and pure Kerr spacetimes. A summary of our results with a concluding discussion and the prospects of future work is presented in Sec. VA and V B. Finally, in the appendix we review the connection between kludge waveforms and the linearized Einstein equations.

Throughout this paper, we will use a system of units in which $G=c=1$. We will denote spacetime indices with Greek letters and space indices with Latin letters.

\section{WAVEFORMS FROM EMRIS IN NONVACUUM SPACETIMES}

Let us consider a curved, nonvacuum spacetime with metric $\boldsymbol{g}$ and with a characteristic length scale $M$ (for a spacetime containing a $\mathrm{SMBH}$, this scale clearly coincides with the black hole mass). The spacetime is intrinsically not a vacuum one because it contains a fluid with a stressenergy tensor $\boldsymbol{T}^{\text {fluid }}$. In addition, consider the presence of a small body, such as a black hole with mass $m \ll M .^{2}$ The small body will of course perturb the geometry of spacetime: the metric $\tilde{\boldsymbol{g}}$ of the physical spacetime can therefore be written as the background metric $\boldsymbol{g}$ plus some perturbations ${ }_{1} \boldsymbol{h}$ of order $O(m / M),{ }_{2} \boldsymbol{h}$ of order $O\left((m / M)^{2}\right)$, etc.:

$$
\tilde{g}_{\mu \nu}=g_{\mu \nu}+{ }_{1} h_{\mu \nu}+{ }_{2} h_{\mu \nu}+O\left((m / M)^{3}\right) .
$$

Similarly, the small body will excite perturbations in the background fluid: the perturbed stress-energy tensor of the fluid can be written as

$$
\tilde{T}_{\mu \nu}^{\text {fluid }}=T_{\mu \nu}^{\text {fluid }}+{ }_{1} \delta T_{\mu \nu}^{\text {fluid }}+{ }_{2} \delta T_{\mu \nu}^{\text {fluid }}+O\left((m / M)^{3}\right) .
$$

In what follows, the background metric $\boldsymbol{g}$ is used to raise and lower tensor indices. For the sake of simplicity, we will also drop the subscript ${ }_{1}$ indicating first-order quantities: in other words, $h_{\mu \nu} \equiv{ }_{1} h_{\mu \nu}$ and $\delta T_{\mu \nu}^{\text {fluid }} \equiv{ }_{1} \delta T_{\mu \nu}^{\text {fluid }}$.

It is well known that the stress-energy tensor of a small body with mass $m$ following a trajectory $z^{\mu}(\tilde{\tau})$ is given by (see for instance Ref. [27])

$$
\tilde{T}_{\text {small body }}^{\alpha \beta}(x)=m \int \tilde{P}_{\mu}^{\alpha}(x, z) \tilde{P}_{\nu}^{\beta}(x, z) \tilde{u}^{\mu} \tilde{u}^{\nu} \frac{\delta^{(4)}(x-z)}{(-\tilde{g})^{1 / 2}} d \tilde{\tau}
$$

where $\tilde{P}_{\mu}^{\alpha}(x, z), \tilde{\tau}$, and $\tilde{u}^{\mu} \equiv d z^{\mu} / d \tilde{\tau}$ are, respectively, the parallel propagator from $z^{\mu}$ to $x^{\mu}$, the proper time, and the 4-velocity in the physical (i.e. perturbed) spacetime. This stress-energy tensor can then be expanded in a series in $m / M$ :

$$
\tilde{T}_{\text {small body }}^{\alpha \beta}=T_{\text {small body }}^{\alpha \beta}+O\left((m / M)^{2}\right),
$$

$$
T_{\text {small body }}^{\alpha \beta}(x)=m \int P_{\mu}^{\alpha}(x, z) P_{\nu}^{\beta}(x, z) u^{\mu} u^{\nu} \frac{\delta^{(4)}(x-z)}{(-g)^{1 / 2}} d \tau,
$$

where $P_{\nu}^{\beta}(x, z), \tau$, and $u^{\mu}=\mathrm{d} z^{\mu} / \mathrm{d} \tau$ are the parallel propagator, proper time, and 4-velocity in the background.

If the small body interacts only gravitationally with the matter contained in the spacetime, its stress-energy tensor is conserved in the physical spacetime:

$$
\tilde{\nabla}_{\beta} \tilde{T}_{\text {small body }}^{\alpha \beta}=0
$$

\footnotetext{
${ }^{2}$ Note that in this context a small black hole can be treated as a small body despite being a singularity of spacetime [26].
} 
( $\tilde{\nabla}$ is the covariant derivative in the physical spacetime). This implies that the small body follows a geodesic of the physical, perturbed spacetime (see for instance Ref. [27] for a formal proof): expanding the geodesic equations in the physical spacetime $\left(\tilde{u}^{\nu} \tilde{\nabla}_{\nu} \tilde{u}^{\mu}=0\right)$ into a series, it is possible to obtain, to first-order in $m / M$,

$$
\begin{aligned}
\frac{D u^{\mu}}{d \tau}= & -\frac{1}{2}\left(g^{\mu \nu}+u^{\mu} u^{\nu}\right)\left(2 \nabla_{\rho} h_{\nu \lambda}-\nabla_{\nu} h_{\lambda \rho}\right) u^{\lambda} u^{\rho} \\
& +O\left((m / M)^{2}\right),
\end{aligned}
$$

where $\nabla$ and $D / d \tau$ are the covariant derivative and the total covariant derivative in the background.

Clearly, to zeroth order Eq. (7) reduces to the geodesic equations in the background spacetime, but it deviates from them at first order. The right-hand side of Eq. (7) represents the so-called "self-force" and is physically due to the interaction of the small body with its own gravitational field $\boldsymbol{h}$; in the case of a small body orbiting around a $\mathrm{SMBH}$, this self-force is responsible for its inspiral towards the black hole.

In order to compute the right-hand side of Eq. (7) one needs to compute the metric perturbation $\boldsymbol{h}$ and because this perturbation is produced by the small body itself, some of its components will be divergent at the position of the small body. A regularization procedure to cure these divergences has been derived $[26,28]$ for the trace-reversed metric perturbations

$$
\bar{h}_{\mu \nu} \equiv h_{\mu \nu}-\frac{1}{2} h_{\alpha}^{\alpha} g_{\mu \nu}
$$

in the Lorenz gauge, which is defined as

$$
\nabla_{\mu} \bar{h}^{\mu \nu}=0 .
$$

It should be noted that while this gauge allows one in principle to remove the problem of divergences and has a number of other advantages (see Ref. [29] for an extensive list), self-force calculations are extremely hard to perform in practice. Indeed, no general inspirals have been computed so far using the regularized version of Eq. (7), not even in Schwarzschild or Kerr spacetimes (see Refs. [27,30] for a review). However, a simpler approach can be followed in which only the dissipative part of the self-force is taken into account, leading to the so-called "adiabatic approximation" [31]. ${ }^{3}$ Within this approximation the small body moves along a geodesic with slowly changing parameters (in Kerr, these parameters are the energy $E$, the angular momentum $L_{z}$, and Carter's constant $Q$ ). One of the advantages of the adiabatic approximation is that it prescribes a way to compute the evolution of these parameters, revealing that their changes $\dot{E}$ and $\dot{L}_{z}$ (with the dot being the derivative with respect to the coordinate time

\footnotetext{
${ }^{3}$ It should be noted that it is not yet clear whether the adiabatic approximation is accurate enough to compute waveforms for LISA as the conservative part of the self-force could have a secular effect as well [32].
}

t) correspond to the energy and angular momentum carried away by gravitational waves [33]. The change in Carter's constant $\dot{Q}$, on the other hand, is harder to compute, although an explicit formula has been recently derived [34].

The first-order metric perturbation $\boldsymbol{h}$ can be computed as a solution of the linearized Einstein equations [35]

$$
\begin{gathered}
\square \bar{h}^{\alpha \beta}+2 R_{\mu}^{\alpha}{ }_{\nu}^{\beta} \bar{h}^{\mu \nu}+S_{\mu}{ }^{\alpha}{ }_{\nu}{ }^{\mu} \bar{h}^{\mu \nu} \\
=-16 \pi\left(\delta T_{\text {fluid }}^{\alpha \beta}+T_{\text {small body }}^{\alpha \beta}\right),
\end{gathered}
$$

where

$$
\begin{gathered}
S_{\mu \alpha \nu \beta}=2 G_{\mu(\alpha} g_{\beta) \nu}-R_{\mu \nu} g_{\alpha \beta}-2 g_{\mu \nu} G_{\alpha \beta}, \\
\square \equiv g^{\mu \nu} \nabla_{\mu} \nabla_{\nu}
\end{gathered}
$$

$\left(R_{\mu \nu \alpha \beta}, R_{\mu \nu}\right.$, and $G_{\mu \nu}$ are the background Riemann, Ricci, and Einstein tensors). Note that self-force effects are not contained in (10), which is a first-order equation. In fact, since the stress-energy tensor of the small body at the lowest order, $\boldsymbol{T}_{\text {small body }}$, is an intrinsically first-order quantity [remember the factor $m$ appearing in Eq. (5)], the small body's contribution can be computed using a zeroth-order expression of $u^{\mu}$ or, equivalently, by solving the geodesic equations for the background metric. In addition to the calculation of the small body's contribution, a consistent solution at first order for the EMRI problem in a curved and nonvacuum spacetime requires the solution of the fluid perturbation $\delta \boldsymbol{T}_{\text {fluid }}$. This can be computed by imposing the conservation of the stress-energy tensor of the fluid, $\tilde{\nabla}_{\beta} \tilde{T}_{\text {fluid }}^{\alpha \beta}=0$, which gives, to first order,

$$
\begin{aligned}
-16 \pi \nabla_{\beta} \delta T_{\text {fluid }}^{\alpha \beta}= & 2 G^{\beta \sigma} \nabla_{\sigma} \bar{h}_{\beta}^{\alpha}-2 G^{\alpha \beta} \partial_{\beta} \bar{h} \\
& -R^{\beta \sigma} \nabla_{\gamma} \bar{h}_{\beta \sigma} g^{\gamma \alpha} .
\end{aligned}
$$

It is not difficult to realize, using Eqs. (10) and (13), that the Lorenz gauge condition is conserved since it satisfies a homogeneous equation

$$
\square\left(\nabla_{\beta} \bar{h}^{\alpha \beta}\right)+R_{\mu}^{\alpha} \nabla_{\beta} \bar{h}^{\mu \beta}=0 .
$$

To summarize, the solution of Eqs. (10) with the right-hand side given by Eqs. (13) and the zeroth-order contribution of Eq. (7), provides the complete and consistent solution of the EMRI problem at first order in $m / M$. Unfortunately, for situations of practical interest, such as for the observations of EMRIs performed by LISA, these first-order waveforms would be sufficiently accurate only for a few days or weeks [21,36], imposing, at least in principle, the need for the solution of second-order equations.

Clearly, the solution of the second-order perturbation equations is much harder to obtain as these will have a schematic generic form of the type

$$
\mathcal{D}\left[{ }_{2} \boldsymbol{h}\right]=O(\nabla \boldsymbol{h} \nabla \boldsymbol{h}, \boldsymbol{h} \nabla \nabla \boldsymbol{h}),
$$

where $\mathcal{D}\left[{ }_{2} \boldsymbol{h}\right]$ is a differential operator acting on ${ }_{2} \boldsymbol{h}$. 
One could naively try to solve this equation by imposing a gauge condition on ${ }_{2} \boldsymbol{h}$ and using the Green function of the $\mathcal{D}$ operator, but the formal solution obtained in this way would be divergent at every point because of the divergences of the first-order perturbation $\boldsymbol{h}$ at the small body's position. A regularization procedure to cure these divergences is known [37], but it has not yet been applied in practical calculations.

An alternative to the solution of the full second-order perturbation equations entails introducing the deviations from geodetic motion in the right-hand side of Eq. (10). This approach is clearly not consistent, but hopefully accurate enough if the ratio $m / M$ and consequently the deviations from geodetic motion are sufficiently small. This is indeed what was done by Drasco and Hughes [38], who used the adiabatic approximation and a simplified formula for $\dot{Q}$ to compute the deviations from geodetic motion, inserting them in the right-hand side of the Teukolsky equation [39] and then solving for first-order perturbations.

While very appealing, as it provides a simple way to improve upon a purely first-order calculation, we will not follow this approach here. Rather, we will perform our calculations within a semirelativistic (kludge) approximation to Eq. (10), using however as a background spacetime a nontrivial departure from a pure Kerr solution. The properties of this spacetime and of alternative formulations of non-Kerr spacetimes will be discussed in detail in Sec. III, while a brief description of our semirelativistic approach will be presented in Sec. IV.

\section{MODELLING THE BACKGROUND SPACETIME}

The discussion made in the previous section assumes that a background spacetime $g$ is known and this is traditionally assumed to be a "pure Kerr" solution. However, this is not the only possibility. Indeed, in order to investigate LISA's ability to detect deviations from Kerr, a number of attempts have been made recently to replace the Kerr metric with other stationary solutions representing reasonable deviations from a single rotating black hole in vacuum. In what follows we will briefly review these attempts and discuss a novel one based on the use of highly accurate numerical solutions of the Einstein equations for spacetimes containing a black hole and a compact torus (see Sec. III C).

\section{A. Approximate non-Kerr spacetimes}

One first attempt to go beyond a pure Kerr model for the central massive object was suggested by Ryan [18-20], who considered a general stationary, axisymmetric, asymptotically flat, vacuum spacetime, which can be used to describe the gravitational field around a central distribution of matter, and its expansion in terms of the mass multipoles $M_{\ell}$ and of the current multipoles $S_{\ell}$ [40].
If one assumes reflection symmetry, the odd $M$-moments and even $S$-moments are identically zero [41,42], so that the nonvanishing moments are the mass $M_{0}=M$, the mass quadrupole $M_{2}$, and the higher-order even multipoles $M_{4}$, $M_{6}, \ldots$, as well as the angular momentum $S_{1}=J$, the current octupole $S_{3}$, and the higher-order odd multipoles $S_{5}, S_{7}, \ldots$. The metric can then be written as

$$
\begin{aligned}
d s^{2}= & -e^{\gamma+\delta} d t^{2}+e^{2 \alpha}\left(d r^{2}+r^{2} d \theta^{2}\right) \\
& +e^{\gamma-\delta} r^{2} \sin \theta^{2}(d \phi-\omega d t)^{2},
\end{aligned}
$$

where the potentials $\gamma, \delta, \omega, \alpha$ depend only on $r$ and $\theta$. Each of them can be expanded in terms of the multipole moments: for example

$$
\begin{aligned}
\delta=\sum_{n=0}^{+\infty}-2 & \frac{M_{2 n}}{r^{2 n+1}} P_{2 n}(\cos \theta)+\text { (higher-order terms) } \\
\omega= & \sum_{n=1}^{+\infty}-\frac{2}{2 n-1} \frac{S_{2 n-1}}{r^{2 n+1}} \frac{P_{2 n-1}^{1}(\cos \theta)}{\sin \theta} \\
& + \text { (higher-order terms) }
\end{aligned}
$$

where $P_{2 n}, P_{2 n-1}^{1}$ are the Legendre and the associated Legendre polynomials and where only the lowest-order $1 / r$-dependence of each multipole moment is shown.

The multipoles are related to the interior matter distribution and could in principle be computed by solving the Einstein equations. In the particular case of a Kerr spacetime, all the multipole moments are trivially related to the first two, mass and angular momentum, by the following relation:

$$
M_{\ell}+\mathrm{i} S_{\ell}=M\left(\mathrm{i} \frac{J}{M}\right)^{\ell}
$$

This is the celebrated "no hair" theorem: an (uncharged) stationary black hole is uniquely determined by its mass and spin. Deviations from the Kerr metric can be therefore detected by measuring the mass, spin, and higher-order moments of the central massive object.

While general and very elegant, this approach has serious drawbacks in the strong-field region near the central massive object, which is clearly the most interesting one. In fact, this is the region which will be mapped by LISA and where the spacetime could be significantly different from Kerr. The origins of these drawbacks are rather apparent when looking at Eqs. (16)-(18), which are in practice an expansion in powers of $1 / r$ around a Minkowski spacetime. As a result, an accurate representation of the strong-field region necessarily requires the inclusion of many multipoles.

Another approach to the modelling of a non-Kerr background spacetime was recently suggested by Babak and Glampedakis in Ref. [21], and is based on the use of the Hartle-Thorne metric [43]. This metric describes the 
spacetime outside slowly rotating stars, includes as a special case the Kerr metric at order $O\left(a^{2}\right)$, where $a \equiv J / M^{2}$, and is accurate up to the mass quadrupole moment. In order to isolate the quadrupolar deviation with respect to Kerr, the Hartle-Thorne metric can be rewritten in terms of the parameter $\epsilon$ defined as

$$
Q=Q^{\mathrm{Kerr}}-\epsilon M^{3}, \quad Q^{\mathrm{Kerr}}=-\frac{J^{2}}{M},
$$

where $M, J$, and $Q \equiv M_{2}$ are the mass, the angular momentum, and the mass quadrupole moment, respectively. Since for Kerr $Q=Q^{\text {Kerr }}, \epsilon$ can be used as a lowest-order measure of the deviation of the spacetime from a Kerr solution. The metric expressed in this way can be further rewritten in "quasi-Boyer-Lindquist coordinates," i.e. coordinates reducing to Boyer-Lindquist coordinates if $\epsilon=0$. This procedure then leads to the "quasi-Kerr" metric

$$
\begin{aligned}
g_{\mu \nu}^{\text {quasi-Kerr }}= & g_{\mu \nu}^{\text {Kerr }}+\epsilon h_{\mu \nu}+O\left(a \epsilon, \epsilon^{2}\right) \\
& +O\left(\delta M_{\ell \geq 4}, \delta S_{\ell \geq 3}\right),
\end{aligned}
$$

where $g_{\mu \nu}^{\text {Kerr }}$ is the Kerr metric in Boyer-Lindquist coordinates, $\epsilon h_{\mu \nu}$ is the deviation from it, and $\delta M_{\ell \geq 4}, \delta S_{\ell \geq 3}$ are the deviations of the higher-order multipoles from those of a Kerr spacetime. Stated differently, the quasi-Kerr metric consists of a Kerr solution plus a small difference in the mass quadrupole expressed by the parameter $\epsilon$, while neglecting any deviations from Kerr in the higher-order multipoles $M_{4}, M_{6}, \ldots$, and $S_{3}, S_{5}, \ldots$, etc.

Because this approach does not involve any expansion in powers of $1 / r$, it can be used in the strong-field regions as long as the central massive object is slowly rotating. Furthermore, it has the great advantage of being straightforward to implement, leaving the mass quadrupole parameter $\epsilon$ as the only adjustable one. However, it has the drawback that it does not include any deviations in the multipoles higher than the quadrupole with respect to the multipoles of pure Kerr, which could be important in the strong-field regime.

\section{B. Exact non-Kerr spacetimes}

A different approach to the modelling of the background consists instead of using exact solutions of the Einstein equations: these spacetimes of course behave well in the strong-field regime, since they are not based on any series expansions.

Few attempts have been made in this direction. However, Kesden, Gair, and Kamionkowski [22] considered spacetimes containing nonrotating boson stars and found that the gravitational waves produced by EMRIs look qualitatively different from the pure black hole case. The spherical boson stars they consider are in fact identical to Schwarzschild spacetimes outside their surfaces, making them indistinguishable from black holes during the initial stages of an EMRI. However, for a black hole the event horizon prevents any observations of the inspirals after the final plunge. Because boson stars are horizonless however, many orbits inside the interior are expected if the small body interacts only gravitationally with the scalar field out of which the star is made: the "smoking gun" for a boson star would therefore be that gravitational waves from the inspiral are observed after the plunge. Gravitational waves from such an event could not be interpreted as an inspiral into a black hole with different parameters, because the first part of the inspiral is identical to the usual black hole inspiral.

Another attempt was made by Collins and Hughes in Ref. [23]. The analytical "bumpy black holes" they build are objects that are almost Schwarzschild black holes, but have some multipoles with a "wrong" value. These spacetimes reduce to the usual Schwarzschild black hole spacetimes in a natural way, by sending the "bumpiness" of the black hole to zero, but unfortunately they require naked singularities: although they are not expected to exist in nature, "bumpy black holes" could be useful as "strawmen" to set up null experiments and test deviations from pure Kerr using EMRIs.

\section{A self-gravitating torus around a rotating black hole}

A different and novel approach to the modelling of a background, non-Kerr spacetime is also possible and will be the one adopted in this paper. In particular, we exploit the consistent numerical solution of the full Einstein equations describing a spacetime with an axisymmetric black hole and a compact, self-gravitating torus of comparable mass and spin. These solutions have been recently obtained to great accuracy with a numerical code using spectral methods. In general, the numerical solution will produce a solution of the Einstein equations representing an axisymmetric and stationary spacetime containing a rotating black hole and a constant-density, uniformly rotating torus of adjustable mass and spin. The metric of this nonpure Kerr spacetime in quasi-isotropic (QI) coordinates is generically given by

$$
\begin{aligned}
d s^{2}= & -e^{2 \nu} d t^{2}+r_{\mathrm{QI}}^{2} \sin ^{2} \theta B^{2} e^{-2 \nu}(d \phi-\omega d t)^{2} \\
& +e^{2 \mu}\left(d r_{\mathrm{QI}}^{2}+r_{\mathrm{QI}}^{2} d \theta^{2}\right),
\end{aligned}
$$

where $\nu, \mu, B$, and $\omega$ are functions of the radial quasiisotropic coordinate $r_{\mathrm{QI}}$ and $\theta$. The procedure for obtaining such numerical solutions is described in detail in Ref. [24] and we here provide only a summary of the main ideas.

The entire spacetime outside of the horizon is described by a metric in Weyl-Lewis-Papapetrou coordinates as in Eq. (22) or (16). We fix our coordinates uniquely by stipulating that the first derivatives of the metric functions be continuous at the ring's surface and by choosing the location of the horizon to be a coordinate sphere $r_{\mathrm{QI}}=$ constant $\equiv r_{+, \mathrm{QI}}$. Specifying the boundary conditions 
$B=0, e^{2 \nu}=0$, and $\omega=$ constant on this sphere ensures that it is indeed a black hole horizon. We further assume reflection symmetry with respect to the equatorial plane.

We are interested only in the metric outside of the black hole and determine it using a multidomain spectral method. One of the domains coincides precisely with the interior of the homogeneous, uniformly rotating perfect fluid ring, the boundary of which must be solved for as part of the global problem. This choice is important in order to avoid Gibbs phenomena. The vacuum region (outside the horizon) is divided up into four subdomains with three fixed boundaries separating them. This somewhat arbitrary choice enables us to resolve functions more accurately in the vicinities of both the ring and the black hole according to the scale determined by each object itself. One of the four vacuum domains extends out to infinity and is then compactified. A mapping of each domain onto a square is chosen in such a way as to avoid steep gradients in the functions being solved for.

The Einstein equations together with the specification of asymptotic flatness and the aforementioned boundary conditions provide us with a complete set of equations to be solved for. The metric functions and the function describing the ring's boundary are expanded in terms of a finite number of Chebyshev polynomials. By specifying physical parameters to describe a configuration and demanding that the equations be fulfilled at collocation points on these five domains, we get a nonlinear system of algebraic equations determining the coefficients in the expansion of the functions. We solve this system using a Newton-Raphson method where an existent neighboring solution provides the initial guess (see Ref. [24] for more details and for a discussion of how to obtain the first "initial guess").

Note that throughout this paper, the masses and angular momenta of the black hole, $M_{\mathrm{BH}}$ and $J_{\mathrm{BH}}$, of the torus, $M_{\text {Torus }}$ and $J_{\text {Torus }}$, and of the whole system, $M_{\text {tot }} \equiv M_{\mathrm{BH}}+$ $M_{\text {Torus }}$ and $J_{\text {tot }} \equiv J_{\mathrm{BH}}+J_{\text {Torus }}$, are the "Komar" masses and angular momenta $[44,45]$. We note that the definition of the mass of a single object in general relativity can be quite slippery, especially when this measure is not an asymptotic one. We also recall that while the total Komar mass of the system coincides with the familiar "Arnowitt-Deser-Misner (ADM)" mass [46], other definitions are possible for the single masses of the torus and the black hole. As an example, it is possible to define the "irreducible mass" of the black hole as $M_{\text {irr }} \equiv$
$\left[A_{+} /(16 \pi)\right]^{1 / 2}$, where $A_{+}$is the horizon's area [47], and then define the total mass of the black hole as $M_{\text {hole }} \equiv$ $\left[M_{\text {irr }}^{2}+\left(J_{\mathrm{BH}} /\left(2 M_{\mathrm{irr}}\right)\right)^{2}\right]^{1 / 2}$ (note that this latter definition coincides with the Komar mass for an isolated Kerr black hole). Similarly, the mass of the torus can also be measured in terms of the "baryonic" mass $M_{\text {baryon }}=\int \rho u^{t} \sqrt{-g} d^{3} x$ ( $\rho$ being the baryonic mass density and $\boldsymbol{u}$ the 4-velocity of the fluid of the torus). This mass is simply a measure of the number of baryons, it does not include the gravitational binding of the object, and thus can also be rather different from the corresponding Komar mass.

Overall, we have found that even in our nonpure Kerr spacetimes, all the definitions of the mass of the black hole agree rather well. In particular, in the spacetimes $A$ and $B$ we will consider in Secs. VA and V B (cf. Table I) we have $M_{\mathrm{BH}}=0.413, M_{\text {irr }}=0.457, M_{\text {hole }}=0.468$, and $M_{\mathrm{BH}}=$ $0.1, M_{\text {irr }}=0.1007, M_{\text {hole }}=0.1007$, respectively. On the other hand, the Komar mass and the baryonic mass of the torus have been found to be different with $M_{\text {Torus }}=0.121$ and $M_{\text {baryon }}=0.0578$ in spacetime $A$ and $M_{\text {Torus }}=0.007$ and $M_{\text {baryon }}=0.00656$ in spacetime $B$. As mentioned above, there is no reason to expect these two measures to be close and it is only interesting that this happens under certain circumstances (as in spacetime $B$, for example). Furthermore, these differences are not going to affect our analysis, which will never use the single mass of the torus.

\section{THE SEMIRELATIVISTIC APPROACH}

Although the procedure outlined in Sec. II to calculate the waveforms from an EMRI in a nonvacuum spacetime is the only mathematically correct one, it has never been applied in practice, not even to first order. Such an approach, in fact, involves the solution of a complicated system of 14 coupled partial differential equations [Eqs. (10) and (13)] and while this can in principle be solved, alternative solutions have been traditionally sought in the literature. A very popular one is the "semirelativistic" approach, which leads to the so-called kludge waveforms [48-50] and which we will also adopt hereafter.

In essence, the semirelativistic approach consists in considering geodetic motion for the small body (including, when possible, corrections to account at least approximately for the effects of radiation reaction) and in calculating the emitted gravitational waves as if the small body were moving in a Minkowski spacetime. This latter as-

TABLE I. Parameters of the spacetimes analyzed in Sec. V, in units in which $10^{7} M_{\odot}=G=c=1 . r_{\text {in, QI }}$ and $r_{\text {out,QI }}$ are the inner and outer edges of the torus in QI coordinates, $\rho$ is the baryonic mass density of the torus and the parameter $\epsilon$ provides a lowest-order measure of the deviation of the spacetime away from a Kerr solution [cf. Eq. (20)]. Note that $\epsilon$ is more sensitive to the ratio between the angular momenta than to that between the masses.

\begin{tabular}{|c|c|c|c|c|c|c|c|c|c|c|c|c|c|}
\hline Spac & $M_{\mathrm{BH}}$ & $M_{\text {Torus }}$ & $M_{\mathrm{BH}} / M_{\text {Torus }}$ & $J_{\mathrm{BH}}$ & $J_{\text {Torus }}$ & $J_{\mathrm{BH}} / J_{\text {Torus }}$ & $J_{\mathrm{BH}} / M_{\mathrm{BH}}^{2}$ & $J_{\text {tot }} / M_{\text {tot }}^{2}$ & $\rho$ & $r_{+, \mathrm{QI}}$ & $r_{\mathrm{in}, \mathrm{QI}}$ & $r_{\text {out, QI }}$ & $\epsilon$ \\
\hline & 413 & 10 & 3 & 3 & 7 & $.69 e-1$ & e & 0.72 & 7 & 0.179 & 64 & 305 & 0.11 \\
\hline$B$ & 0.100 & 0.007 & 14.3 & $-1.74 \mathrm{e}-5$ & $2.58 \mathrm{e}-3$ & $-6.74 \mathrm{e}-3$ & $-1.74 \mathrm{e}-3$ & 0.224 & 0.198 & 0.050 & 0.9156 & 1.0000 & 2.63 \\
\hline
\end{tabular}


sumption requires a mapping between the real spacetime and the Minkowski spacetime: in the pure Kerr case, this is obtained by identifying Boyer-Lindquist coordinates with the spherical coordinates of the Minkowski spacetime. The waveforms are then computed using the standard quadrupole, octupole, or higher-order formulas. ${ }^{4}$ Waveforms obtained in this way are commonly referred to as kludge waveforms [48-50]

A justification of this procedure in terms of the Einstein equations is given in the appendix. However, the strongest motivation for introducing kludge waveforms is the surprising agreement they show with the accurate waveforms that can be computed in a Kerr spacetime using the Teukolsky formalism [50]. In view of this, kludge waveforms represent the natural first approach to model the emission from EMRIs in nonpure Kerr spacetimes and will be used throughout this work.

As mentioned in Sec. II, the adiabatic approximation offers a simple way to include radiation-reaction effects in a Kerr spacetime. More specifically, if we denote Kerr geodesics by $x_{\text {geod }}^{\mu}\left(t, E, L_{z}, Q\right)$, the trajectory of the small body is then corrected to be $x^{\mu}(t)=x_{\text {geod }}^{\mu}\left(t, E(t), L_{z}(t)\right.$, $Q(t))$, that is, a geodesic with varying parameters. The accurate calculation of the fluxes $\dot{E}, \dot{L}_{z}$, and $\dot{Q}$ is rather involved [34,38], but approximate ways to compute them have also been suggested [51-53]. Although Barack and Cutler [25] have recently proposed including radiation reaction in quasi-Kerr spacetimes by using postNewtonian fluxes in which the leading-order effect of the quadrupole of the spacetime is taken into account, it is still unclear at this stage how accurately the fluxes for a Kerr or a quasi-Kerr spacetime could describe the nongeodetic motion of an EMRI around our black hole-torus systems. Because of this, we have here preferred to consider the simplest scenario and thus model the motion of the small body as a pure geodesic with equations of motion that in the spacetime Eq. (22) are given by

$$
\begin{gathered}
\frac{d t}{d \tau}=-g^{t t} \tilde{\boldsymbol{\epsilon}}+g^{t \phi} \tilde{\ell}, \\
\frac{d \phi}{d \tau}=-g^{t \phi} \tilde{\boldsymbol{\epsilon}}+g^{\phi \phi} \tilde{\ell}, \\
\frac{d^{2} r}{d \tau^{2}}=-\Gamma_{r r}^{r}\left(\frac{d r}{d \tau}\right)^{2}-\Gamma_{\theta \theta}^{r}\left(\frac{d \theta}{d \tau}\right)^{2}-2 \Gamma_{r \theta}^{r} \frac{d r}{d \tau} \frac{d \theta}{d \tau} \\
-\Gamma_{t t}^{r}\left(\frac{d t}{d \tau}\right)^{2}-\Gamma_{\phi \phi}^{r}\left(\frac{d \phi}{d \tau}\right)^{2}-2 \Gamma_{t \phi}^{r} \frac{d t}{d \tau} \frac{d \phi}{d \tau},
\end{gathered}
$$

\footnotetext{
${ }^{4}$ Note that comparisons with Teukolsky-based waveforms in Kerr show that the inclusion of multipoles higher than the octupole does not improve kludge waveforms [50]. Because of this, contributions only up to the octupole are used here to calculate kludge waveforms.
}

$$
\begin{aligned}
\frac{d^{2} \theta}{d \tau^{2}}= & -\Gamma_{r r}^{\theta}\left(\frac{d r}{d \tau}\right)^{2}-\Gamma_{\theta \theta}^{\theta}\left(\frac{d \theta}{d \tau}\right)^{2}-2 \Gamma_{r \theta}^{\theta} \frac{d r}{d \tau} \frac{d \theta}{d \tau} \\
& -\Gamma_{t t}^{\theta}\left(\frac{d t}{d \tau}\right)^{2}-\Gamma_{\phi \phi}^{\theta}\left(\frac{d \phi}{d \tau}\right)^{2}-2 \Gamma_{t \phi}^{\theta} \frac{d t}{d \tau} \frac{d \phi}{d \tau},
\end{aligned}
$$

where $r \equiv r_{\mathrm{QI}}$ is the radial quasi-isotropic coordinate, $\tau$ is the proper time, the $\Gamma$ 's are the Christoffel symbols, and $\tilde{\epsilon}$ and $\tilde{\ell}$ are the energy and angular momentum per unit mass as measured by an observer at infinity.

The resulting geodesics can be labeled with seven parameters: four refer to the initial position $t_{0}, \phi_{0}, r_{0}, \theta_{0}$ and the remaining three identify the initial 4-velocity. In the case, which we will consider in this paper, of bound stable orbits in the equatorial plane, only five parameters would remain. However, because of stationarity and axisymmetry it is not restrictive to fix $t_{0}=\phi_{0}=0$ and $r_{0}=r_{p}$, being $r_{p}$ the periastron radius. Therefore, except for a sign to distinguish between prograde $(\dot{\phi}>0)$ and retrograde $(\dot{\phi}<0)$ orbits, equatorial bound stable geodesics can be characterized by two parameters only, which we can choose to be the so-called "latus rectum" $p_{\mathrm{QI}}$ and the "eccentricity" $e_{\mathrm{QI}}$, which are related to the coordinate radii at apoastron and periastron by $r_{a} \equiv p_{\mathrm{QI}} /\left(1-e_{\mathrm{QI}}\right)$ and $r_{p} \equiv p_{\mathrm{QI}} /\left(1+e_{\mathrm{QI}}\right)$.

Clearly, kludge waveforms computed from pure geodetic motion are expected to be accurate only below the time scale over which radiation-reaction effects become apparent and make our waveforms differ significantly from the real signal. A simple way to estimate this time scale exploits the concept of "overlap" between two waveforms, which will be introduced in Sec. IVA.

An important comment needed here is instead on the coordinate mapping used in calculating kludge waveforms. As already mentioned, this mapping has a straightforward realization in a Kerr spacetime, where the BL coordinates are associated with the spherical coordinates of a Minkowski spacetime. In a similar manner, in our nonpure Kerr spacetimes we transform the solution of the geodesic equations from QI coordinates to "quasi-BoyerLindquist" (QBL) coordinates, i.e. coordinates that reduce to $\mathrm{BL}$ coordinates in the absence of the torus. These coordinates are then identified with the spherical coordinates of a Minkowski spacetime as in Ref. [21] and used to compute kludge waveforms.

Fortunately, the transformation from QI to QBL coordinates is straightforward and involves only a change in the radial coordinate:

$$
r_{\mathrm{QBL}}=r_{\mathrm{QI}}+\tilde{M}+\frac{r_{+, \mathrm{QI}}^{2}}{r_{\mathrm{QI}}}
$$

where $\tilde{M}$ is a parameter that reduces to the mass of the central black hole in the absence of the torus. Clearly, this mapping suffers from an intrinsic ambiguity as the mass $\tilde{M}$ could be either associated with the mass of the black hole or with the total mass of the system, or even with a combination of the two. Although all the choices are 


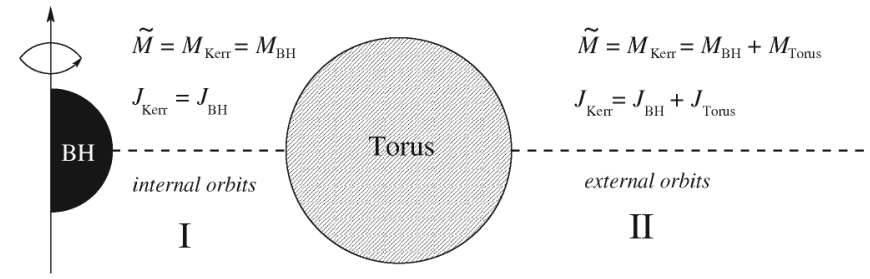

FIG. 1. Schematic classification of the two regions of the spacetime. For equatorial orbits in region I (i.e. internal orbits) the mass and angular momentum of the Kerr black hole coincide with the mass and angular momentum of the black hole. For equatorial orbits in region II (i.e. external orbits) the mass and angular momentum of the Kerr black hole coincide with the total mass and angular momentum of the black hole-torus system.

essentially equivalent when the torus is very light, this is not necessarily the case for some of the configurations considered here, for which the torus has a mass comparable with that of the black hole. Since the parameter $\tilde{M}$ is, at least in a Newtonian sense, the gravitational mass experienced by the small body, we have here followed a pragmatic approach and set $\tilde{M}=M_{\text {tot }}$ for equatorial orbits with periastron larger than the outer edge of the torus, which we will refer to as the "external orbits." Conversely, we have set $\tilde{M}=M_{\mathrm{BH}}$ for what we will refer to as the "internal orbits," that is equatorial orbits with both periastron and apoastron between the inner edge of the torus and the horizon. This classification is summarized schematically in Fig. 1, which shows the two regions into which the spacetime has been divided and the corresponding values of $\tilde{M}$. This choice is clearly an operative ansatz, but we have checked to see that its influence on our results is indeed negligible and a detailed discussion of this will be presented in Sec. V.

Finally, we note that we have not considered orbits crossing the torus because the nongravitational interaction between the small body and the fluid would cause deviations from geodetic motion which are not easy to model.

\section{A. Overlap and dephasing time}

In order to compare (kludge) waveforms computed in nonpure Kerr spacetimes with (kludge) waveforms computed in Kerr spacetimes, we follow the procedure proposed in Ref. [21] and make use of the so-called overlap function. Its meaning can be best understood through the more familiar concept of SNR, which we will now briefly review.

We recall that if a signal $s(t)$ is the sum of a gravitational wave $h(t)$ and of some Gaussian noise $n(t)$, the SNR for a template $\hat{h}(t)$ is given by [54]

$$
\frac{S}{N}[\hat{h}]=\frac{\int \hat{h}(t) w(t-\tau) s(\tau) d \tau d t}{\operatorname{rms}\left[\int \hat{h}(t) w(t-\tau) n(\tau) d \tau d t\right]}=\frac{(\hat{h}, s)}{(\hat{h}, \hat{h})^{1 / 2}}
$$

where $w(t)$ is Wiener's optimal filter (i.e. the Fourier trans- form of the function $w(t)$ is given by $\tilde{w}(f)=1 / S_{n}(f)$, with $S_{n}(f)$ being the spectral sensitivity of the detector), "rms" denotes the root mean square, and the internal product "(,)" can be defined in terms of the Fourier transforms (which are denoted by a "tilde"):

$$
\left(h_{1}, h_{2}\right) \equiv 2 \int_{0}^{\infty} \frac{\tilde{h}_{1}^{*}(f) \tilde{h}_{2}(f)+\tilde{h}_{1}(f) \tilde{h}_{2}^{*}(f)}{S_{n}(f)} d f .
$$

Clearly, the SNR of Eq. (28) is a Gaussian random variable with zero average and unit variance if no gravitational-wave signal is present. On the other hand, in the presence of a gravitational wave the expected value for the SNR is nonzero with a time average given by

$$
\left\langle\frac{S}{N}[\hat{h}]\right\rangle=\frac{(\hat{h}, h)}{(\hat{h}, \hat{h})^{1 / 2}}+\frac{(\hat{h}, n)}{(\hat{h}, \hat{h})^{1 / 2}}=\frac{(\hat{h}, h)}{(\hat{h}, \hat{h})^{1 / 2}} .
$$

If $\alpha$ measures the SNR for a template $\hat{h}(t)$ "matching" the gravitational wave $h(t)$ perfectly, i.e. $\langle S / N\rangle=(h, h)^{1 / 2} \equiv$ $\alpha$, any "mismatch" between $\hat{h}(t)$ and $h(t)$ will degrade the SNR to $\langle S / N\rangle=\alpha \mathcal{O}(h, \hat{h})$, where the overlap function $\mathcal{O}$ is defined as

$$
\mathcal{O}(h, \hat{h}) \equiv \frac{(h, \hat{h})}{(\hat{h}, \hat{h})^{1 / 2}(h, h)^{1 / 2}} .
$$

The same logic can now be used to quantify the differences between kludge waveforms computed in different spacetimes. More specifically, if we label with " 1 " a waveform computed in a nonpure Kerr spacetime and with "2" the closest equivalent in a Kerr spacetime, the overlap between the two $\mathcal{O}\left(h_{1}, h_{2}\right) \equiv$ $\left(h_{1}, h_{2}\right) /\left[\left(h_{1}, h_{2}\right)^{1 / 2}\left(h_{1}, h_{2}\right)^{1 / 2}\right]$ will express how much SNR is lost by an observer match-filtering a black holetorus signal with a pure Kerr template. Stated differently, $\mathcal{O}\left(h_{1}, h_{2}\right)=1$ if the two waveforms are identical, while $\mathcal{O}\left(h_{1}, h_{2}\right)=0$ if they are totally uncorrelated and $\mathcal{O}\left(h_{1}, h_{2}\right)=-1$ if they are perfectly anticorrelated.

Having introduced the concept of overlap function, we can proceed to an operative definition of the time scale below which kludge waveforms computed from pure geodetic motion are expected to be accurate. This time scale, usually referred to as the "dephasing time" $\tau_{\mathrm{d}}$, is defined as the time at which the overlap between two waveforms in the Kerr spacetime, one computed considering geodetic motion and the other one including radiation-reaction effects, drops below 0.95 (this is indeed the threshold used to build template banks [55]). Clearly, the dephasing time will be different for external and internal orbits and also in this case attention needs to be paid to the mappings between nonpure and pure Kerr spacetimes. Following the same logic discussed in the previous section, we calculate $\tau_{\mathrm{d}}$ for an external equatorial orbit in our nonpure Kerr spacetime by considering the equatorial orbit with the same latus 
rectum and eccentricity in the Kerr spacetime ${ }^{5}$ with mass $M_{\text {Kerr }}=M_{\text {tot }}$ and spin $J_{\text {Kerr }}=J_{\text {tot }}$. On the other hand, for an internal orbit we calculate $\tau_{\mathrm{d}}$ by considering the orbit with the same latus rectum and eccentricity in the Kerr spacetime with mass $M_{\mathrm{Kerr}}=M_{\mathrm{BH}}$ and spin $J_{\mathrm{Kerr}}=J_{\mathrm{BH}}$. As we will explain, in this case we have also looked into the influence that this association has on the overall results presented in Sec. V.

In order to compute the dephasing time, we used the approximate Kerr fluxes proposed in Ref. [53], which are based on post-Newtonian expansions and fits to fluxes computed rigorously with the Teukolsky formalism.

\section{COMPARING PURE AND NONPURE KERR SPACETIMES}

The set of tools introduced in the previous sections, namely: the kludge waveforms, the numerical solution of the Einstein equations for spacetimes containing a black hole and a torus, and the overlap function, can now be applied to determine to what extent LISA can detect a difference between a pure and a nonpure Kerr spacetime.

Hereafter we will restrict our attention to equatorial, bound, and stable orbits, choosing the values of the mass and angular momentum of the pure Kerr spacetime using the same logic discussed in the previous sections, i.e.

$$
\left.\begin{array}{rl}
M_{\text {Kerr }} & =M_{\mathrm{BH}}=\tilde{M} \\
J_{\mathrm{Kerr}} & =J_{\mathrm{BH}} \\
M_{\mathrm{Kerr}} & =M_{\mathrm{tot}}=\tilde{M} \\
J_{\mathrm{Kerr}} & =J_{\mathrm{tot}}
\end{array}\right\} \text { internal orbits, }
$$

Note that for internal orbits we did try to compare our nonpure Kerr spacetimes with pure Kerr spacetimes having $M_{\text {Kerr }}=M_{\text {tot }}=\tilde{M}$ and $J_{\text {Kerr }}=J_{\text {tot }}$ (using these values also to compute the dephasing time, cf. Sec. ), but this turned out not to be a good choice. ${ }^{6}$

Once a nonpure and a pure Kerr spacetime have been built and the orbits have been isolated according to the relations (32), further care needs to be paid in selecting corresponding geodesics. As mentioned in Sec. IV, equatorial geodesics can be labeled by two parameters, which can be chosen to be, for instance, the latus rectum and the eccentricity $p_{(\mathrm{Q}) \mathrm{BL}}$ and $e_{(\mathrm{Q}) \mathrm{BL}}$, calculated in QBL coordinates for the nonpure Kerr spacetime and in BL coordinates for the Kerr spacetime.

However, as already pointed out in Ref. [21], waveforms produced by geodesics having the same $p_{(\mathrm{Q}) \mathrm{BL}}$ and $e_{(\mathrm{Q}) \mathrm{BL}}$

\footnotetext{
${ }^{5}$ The latus rectum and the eccentricity are assumed to be in $\mathrm{BL}$ coordinates in pure Kerr and in QBL in nonpure Kerr spacetimes.

${ }^{6}$ i.e., for many bound stable orbits in the nonpure Kerr spacetimes that we considered, it was impossible even to find bound stable orbits with the same latus rectum and eccentricity in the Kerr spacetime.
}

are significantly different because they do not contain comparable orbital frequencies, and give overlaps $\mathcal{O} \leq$ 0.4 . A similar conclusion can be drawn in the case in which the free parameters are chosen to be the periastron radius and the (tangential) velocity measured at the periastron by a zero angular momentum observer (ZAMO): this choice gives overlaps $\mathcal{O} \simeq 0.1-0.2$. In view of this, any sensible comparison can be made only with geodesics in the two spacetimes that have the same orbital frequencies (this result was already pointed out in Ref. [21]).

We recall that an equatorial geodesic in a generic stationary, axisymmetric spacetime has an $r$-motion that is periodic in the coordinate time $t$. To see this, it is sufficient to combine Eqs. (23) and (24) and the normalization condition $u_{\mu} u^{\mu}=-1$ for an equatorial motion $\theta=\pi / 2$ so that

$$
(d r / d t)^{2}=V(r, \tilde{\epsilon}, \tilde{\ell})
$$

with $V(r, \tilde{\epsilon}, \tilde{\ell})$ being a function of $r$ and of the two constants of motion $\tilde{\epsilon}$ and $\tilde{\ell}$. Clearly, Eq. (33) has a periodic solution with a frequency that we will denote $\omega_{r}$. A similar analysis can be carried out for the motion in the $\phi$ direction, which, combining Eqs. (23) and (24) with $\theta=\pi / 2$, satisfies an equation of the type

$$
d \phi / d t=G(r, \tilde{\epsilon}, \tilde{\ell})
$$

where $G(r, \tilde{\epsilon}, \tilde{\ell})$ is again a function of $r, \tilde{\epsilon}$, and $\tilde{\ell}$. Integrating Eq. (34) with $\phi_{0}=t_{0}=0$ leads to

$$
\phi(t)=\langle G\rangle t+\int_{0}^{t}(G(r(t), \tilde{\epsilon}, \tilde{\ell})-\langle G\rangle) d t,
$$

where $\langle G\rangle$ is the time average of $G(r(t), \tilde{\epsilon}, \tilde{\ell})$ over an $r$-period. The second term on the right-hand side of Eq. (35) is clearly periodic (with zero average) in $t$ so that the $\phi$-motion has a linearly growing term and an oscillating one. The overall frequency content of the $\phi$ motion is therefore determined by $\omega_{\phi}=\langle G\rangle$.

The orbital frequencies $\omega_{r}$ and $\omega_{\phi}$ can therefore be used to characterize equatorial geodesics (and hence the waveforms) that are expected to be as similar as possible (i.e. have the largest possible overlap) in the two spacetimes. In practice, given a geodesic (and therefore a waveform) characterized by $\omega_{r}$ and $\omega_{\phi}$ in the nonpure Kerr spacetime, we can compare it to the waveform produced in the Kerr spacetime by the orbit which has the same $r$ - and $\phi$-frequencies. Since $\omega_{r}$ and $\omega_{\phi}$ for equatorial orbits in a Kerr spacetime are functions of $M_{\mathrm{Kerr}}, J_{\mathrm{Kerr}}, p_{\mathrm{BL}}$, and $e_{\mathrm{BL}}$ (explicit expressions for these functions, which we will denote $\omega_{r}^{\text {Kerr }}$ and $\omega_{\phi}^{\text {Kerr }}$, are given in Ref. [56]), matching the geodesics amounts to solving the following equations in the unknowns $\delta p$ and $\delta e$ 


$$
\begin{aligned}
& \omega_{r}^{\mathrm{BH}+\operatorname{Torus}}\left(p_{\mathrm{QBL}}, e_{\mathrm{QBL}}\right)=\omega_{r}^{\mathrm{Kerr}}\left(p_{\mathrm{BL}}=p_{\mathrm{QBL}}+\delta p, e_{\mathrm{BL}}=e_{\mathrm{QBL}}+\delta e, M_{\mathrm{Kerr}}, J_{\mathrm{Kerr}}\right), \\
& \omega_{\phi}^{\mathrm{BH}+\operatorname{Torus}}\left(p_{\mathrm{QBL}}, e_{\mathrm{QBL}}\right)=\omega_{\phi}^{\mathrm{Kerr}}\left(p_{\mathrm{BL}}=p_{\mathrm{QBL}}+\delta p, e_{\mathrm{BL}}=e_{\mathrm{QBL}}+\delta e, M_{\mathrm{Kerr}}, J_{\mathrm{Kerr}}\right),
\end{aligned}
$$

where $\omega_{r}^{\mathrm{BH}+\mathrm{Torus}}\left(p_{\mathrm{QBL}}, e_{\mathrm{QBL}}\right)$ and $\omega_{\phi}^{\mathrm{BH}+\mathrm{Torus}}\left(p_{\mathrm{QBL}}, e_{\mathrm{QBL}}\right)$ are the $r$ - and $\phi$-frequencies of the equatorial orbit with latus rectum $p_{\mathrm{QBL}}$ and eccentricity $e_{\mathrm{QBL}}$ in the nonpure Kerr spacetime under consideration and where $M_{\text {Kerr }}, J_{\text {Kerr }}$ follow the selection rule in Eq. (32) to distinguish internal and external orbits. Indeed, this is the approach which was followed in Ref. [21] and which highlighted the possibility of a confusion problem in nonpure Kerr spacetimes.

An important difference with respect to the work presented in Ref. [21] is that we also considered a different way in which it is possible to identify geodesics that have the same orbital frequencies in a Kerr and in a nonpure Kerr spacetime. We can in fact consider the latus rectum and eccentricity fixed in (Q)BL and search for the values of the additional mass $\delta M$ and angular momentum $\delta J$ of the Kerr spacetime which would yield the same $r$ - and $\phi$-frequencies, i.e.

$$
\begin{aligned}
& \omega_{r}^{\mathrm{BH}+\text { Torus }}\left(p_{\mathrm{QBL}}, e_{\mathrm{QBL}}\right)=\omega_{r}^{\mathrm{Kerr}}\left(p_{\mathrm{BL}}=p_{\mathrm{QBL}}, e_{\mathrm{BL}}=e_{\mathrm{QBL}}, M_{\mathrm{Kerr}}+\delta M, J_{\mathrm{Kerr}}+\delta J\right) \text {, } \\
& \omega_{\phi}^{\mathrm{BH}+\text { Torus }}\left(p_{\mathrm{QBL}}, e_{\mathrm{QBL}}\right)=\omega_{\phi}^{\mathrm{Kerr}}\left(p_{\mathrm{BL}}=p_{\mathrm{QBL}}, e_{\mathrm{BL}}=e_{\mathrm{QBL}}, M_{\mathrm{Kerr}}+\delta M, J_{\mathrm{Kerr}}+\delta J\right) \text {. }
\end{aligned}
$$

Of course, a similar but distinct set of equations can also be built by considering orbits having the same latus rectum and eccentricity in QI coordinates ${ }^{7}$

$$
\begin{aligned}
& \omega_{r}^{\mathrm{BH}+\operatorname{Torus}}\left(p_{\mathrm{QI}}, e_{\mathrm{QI}}\right)=\omega_{r}^{\mathrm{Kerr}}\left(p_{\mathrm{QI}}, e_{\mathrm{Q}}, M_{\mathrm{Kerr}}+\delta M, J_{\text {Kerr }}+\delta J\right), \\
& \omega_{\phi}^{\mathrm{BH}+\operatorname{Torus}}\left(p_{\mathrm{QI}}, e_{\mathrm{QI}}\right)=\omega_{\phi}^{\mathrm{Kerr}}\left(p_{\mathrm{QI}}, e_{\mathrm{Q}}, M_{\mathrm{Kerr}}+\delta M, J_{\text {Kerr }}+\delta J\right) .
\end{aligned}
$$

To illustrate how different correlations of orbits in the two spacetimes can lead to significantly different waveforms, we show in Fig. 2 some kludge waveforms for a small body with mass $m=1 M_{\odot}$ moving in the spacetime $B$ whose parameters are listed in Table I. The geodesics

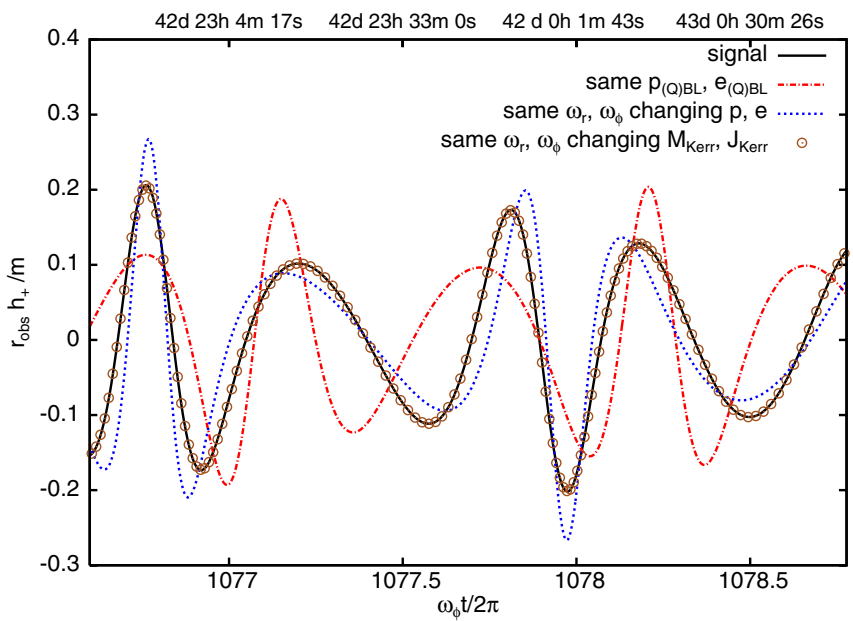

FIG. 2 (color online). Kludge waveforms around the dephasing time for a small body with mass $m=1 M_{\odot}$ moving in the spacetime $B$ of Table I. The solid line shows the waveform produced by a geodesic with given latus rectum and eccentricity in spacetime $B$, while the dot-dashed line refers to a geodesic with the same latus rectum and eccentricity (in (Q)BL coordinates) in a Kerr spacetime with $M_{\text {Kerr }}=M_{\text {tot }}$ and $J_{\text {Kerr }}=J_{\text {tot }}$. The dotted line and the circles are instead the waveforms produced by an orbit with the same $r$ - and $\phi$-frequencies as obtained by adjusting $(\delta p, \delta e)$ or $(\delta M, \delta J)$, respectively. have been calculated up to the dephasing time (i.e. $\tau_{\mathrm{d}} \simeq$ $42 \mathrm{~d}$ ) and the figure shows a magnification of the waveforms around this time. In particular, the solid line shows the waveform produced by a geodesic with $p_{\mathrm{QBL}} / M_{\mathrm{tot}}=$ 21.237 and $e_{\mathrm{QBL}}=0.212$ in spacetime $B$, while the dotdashed line refers to a geodesic with the same latus rectum and eccentricity (in (Q)BL coordinates) in a Kerr spacetime with $M_{\text {Kerr }}=M_{\text {tot }}$ and $J_{\text {Kerr }}=J_{\text {tot }}$. The dotted line and the circles are instead the waveforms produced by an orbit with the same $r$ - and $\phi$-frequencies as obtained by adjusting $(\delta p, \delta e)$ or $(\delta M, \delta J)$, respectively. Clearly, fixing the same orbital parameters $p_{(\mathrm{Q}) \mathrm{BL}}$ and $e_{(\mathrm{Q}) \mathrm{BL}}$ in the two spacetimes would be misleading and will inevitably produce very small overlaps. On the other hand, ensuring that the orbital frequencies are the same by adjusting $\delta M$ and $\delta J$ provides waveforms that are much more similar and even harder to distinguish over this time scale than if $\delta p$ and $\delta e$ are adjusted.

In the following sections we will discuss in detail the confusion problem when considering the two different ways in which the geodesics in the two spacetimes can be matched. Before doing that, however, we will now briefly recall the main properties of the numerically generated spacetimes that we have considered here, and whose parameters are listed in Table I. We note that because the

\footnotetext{
${ }^{7}$ The transformation from BL to QI coordinates in a Kerr spacetime is given for instance in Ref. [57], Eq. (80): the transformation turns out to be the inverse of Eq. (27), with $r_{+\mathrm{QI}}=$ $M\left(1-a^{2}\right)^{1 / 2} / 2(M$ and $a$ being the mass and the spin parameter of the Kerr spacetime under consideration).
} 
investigation of each spacetime is a rather lengthy and computationally expensive operation, we have restricted our attention to two spacetimes only, but with rather different properties. More specifically, we have considered a first spacetime (denoted as $A$ ) having a torus with mass comparable with that of the black hole and slightly larger angular momentum (i.e. $M_{\mathrm{BH}} \gtrsim M_{\text {Torus }},\left|J_{\mathrm{BH}}\right| \lesssim\left|J_{\text {Torus }}\right|$ ) and a second spacetime (denoted as $B$ ) having a torus with mass much smaller than that of the black hole but much larger angular momentum (i.e. $M_{\mathrm{BH}} \gg M_{\text {Torus }}$, $\left.\left|J_{\mathrm{BH}}\right| \ll\left|J_{\text {Torus }}\right|\right)$.

We also note that spacetime $A$ has a rather small quadrupole parameter $\epsilon \simeq 0.1$ [cf. Eq. (20) for the definition] and could therefore be used to validate the perturbative results of Ref. [21] which, we recall, were formulated to the lowest order in $\epsilon$. Interestingly, we will see that taking into account the higher-order multipoles can lead to important qualitative differences and weaken or even cancel, for orbits very close to the torus, the confusion problem found in Ref. [21]. Spacetime $B$, on the other hand, has a considerably larger value for $\epsilon$ and cannot, therefore, be described satisfactorily by the metric (21). The spacetimes were computed to sufficiently high accuracy so as to ensure that the numerical errors do not affect the results. More specifically, for spacetime $A$ we used $40 \times 40$ Chebyshev polynomials in the vacuum domain extending out to infinity (domain 1 of [24]) and $28 \times 28$ polynomials in the other 4 domains. For spacetime $B$ we used $31 \times 27$ polynomials in each of the 5 domains. Typical physical quantities, such as mass and angular momentum, were thus accurate to about $10^{-6}$ in spacetime $A$ and $10^{-7}$ in spacetime $B$. Besides these being errors that are orders of magnitude smaller than the ones $O\left(a \epsilon, \epsilon^{2}\right)+O\left(\delta M_{\ell \geq 4}, \delta S_{\ell \geq 3}\right)$ typically affecting the approximate metric (21), the accuracy of our numerically generated spacetimes is sufficient for our purposes, since the dephasing it introduces is comparable with the dephasing due to radiation reaction, as the latter scales with the mass ratio $m / M_{\mathrm{BH}} \approx 10^{-6}-10^{-7}$. As a result, introducing a cutoff at the dephasing time not only makes the effects of radiation reaction negligible, but it also ensures that the numerical errors in the calculation of the spacetimes do not affect the results. As a further check, we have varied the number of Chebyshev polynomials and verified that the numerical errors inherent to the spacetimes have a negligible impact on our final results.

For all of the waveforms computed in this paper, we have considered an observer located at $\phi_{\mathrm{obs}}=0, \theta_{\mathrm{obs}}=$ $\pi / 4$ and decomposed the incoming gravitational-wave signal into the usual "plus" and "cross" polarizations (see, for instance, Refs. [50,58] for details). Furthermore, labelling the gravitational waves computed in the two spacetimes with 1 and 2, we calculate the overlap between both polarizations, $\mathcal{O}\left(h_{1}^{+}, h_{2}^{+}\right)$and $\mathcal{O}\left(h_{1}^{\times}, h_{2}^{\times}\right)$, and in the discussion of our results we refer to the smallest of the two overlaps, i.e. $\mathcal{O}\left(h_{1}, h_{2}\right) \equiv \min \left[\mathcal{O}\left(h_{1}^{+}, h_{2}^{+}\right), \mathcal{O}\left(h_{1}^{\times}, h_{2}^{\times}\right)\right]$. In practice, however, the difference between $\mathcal{O}\left(h_{1}^{+}, h_{2}^{+}\right)$and

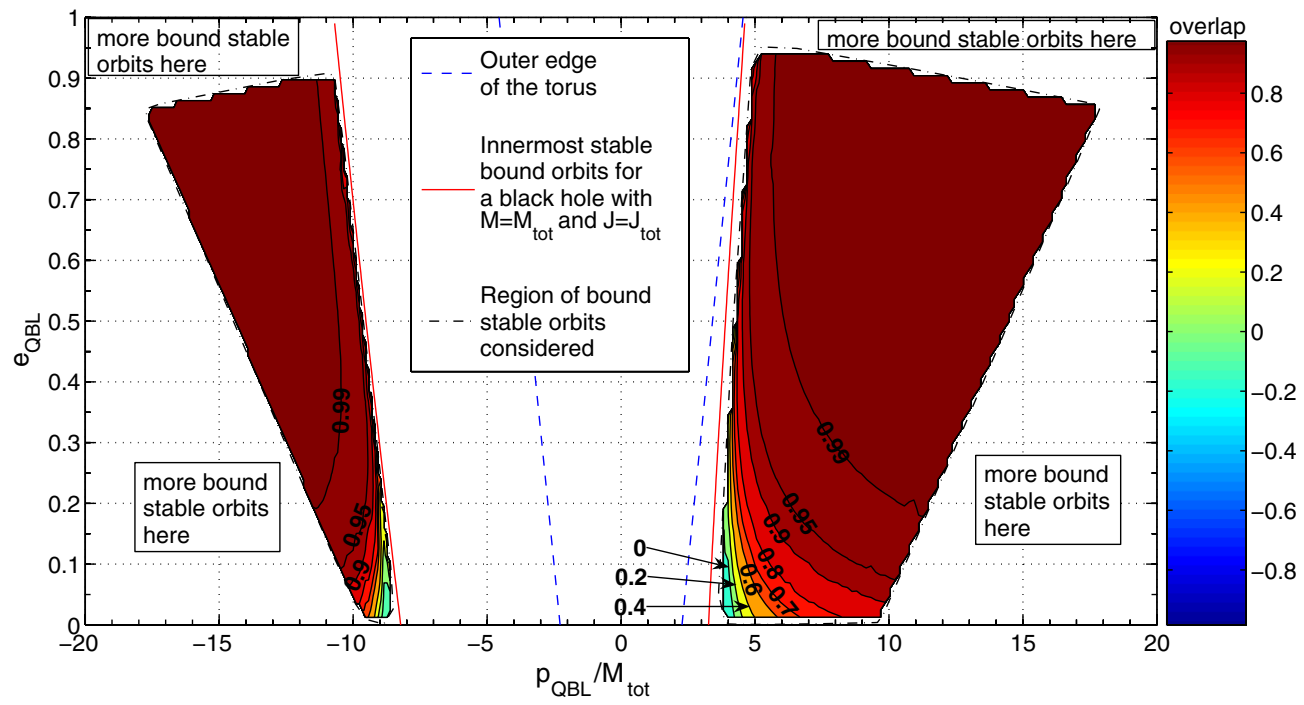

FIG. 3 (color online). Overlap between waveforms produced in spacetime $A$ by external orbits and waveforms produced in a Kerr spacetime with mass $M_{\text {Kerr }}=M_{\text {tot }}$ and spin $J_{\text {Kerr }}=J_{\text {tot }}$. The orbits all have the same $r$ - and $\phi$-frequencies as obtained by suitably changing the latus rectum and the eccentricity, with positive values of $p_{\mathrm{QBL}}$ referring to prograde orbits, and negative ones to retrograde orbits. The different lines mark the margins of the different relevant regions of the $\left(p_{\mathrm{QBL}}, e_{\mathrm{QBL}}\right)$ plane, with the dashed line representing the outer "edge of the torus," the solid line representing the innermost stable bound orbits for a Kerr spacetime with mass $M_{\mathrm{Kerr}}=M_{\mathrm{tot}}$ and spin $J_{\mathrm{Kerr}}=J_{\mathrm{tot}}$, and the dot-dashed line limiting the regions of the $\left(p_{\mathrm{QBL}}, e_{\mathrm{QBL}}\right)$ plane where bound stable orbits have been studied. A high overlap in large regions of the space of parameters indicates that a confusion problem is indeed possible in this spacetime for observational time scales below or comparable to the dephasing time, although this confusion disappears for orbits with small eccentricities and close to the innermost bound stable orbits. 


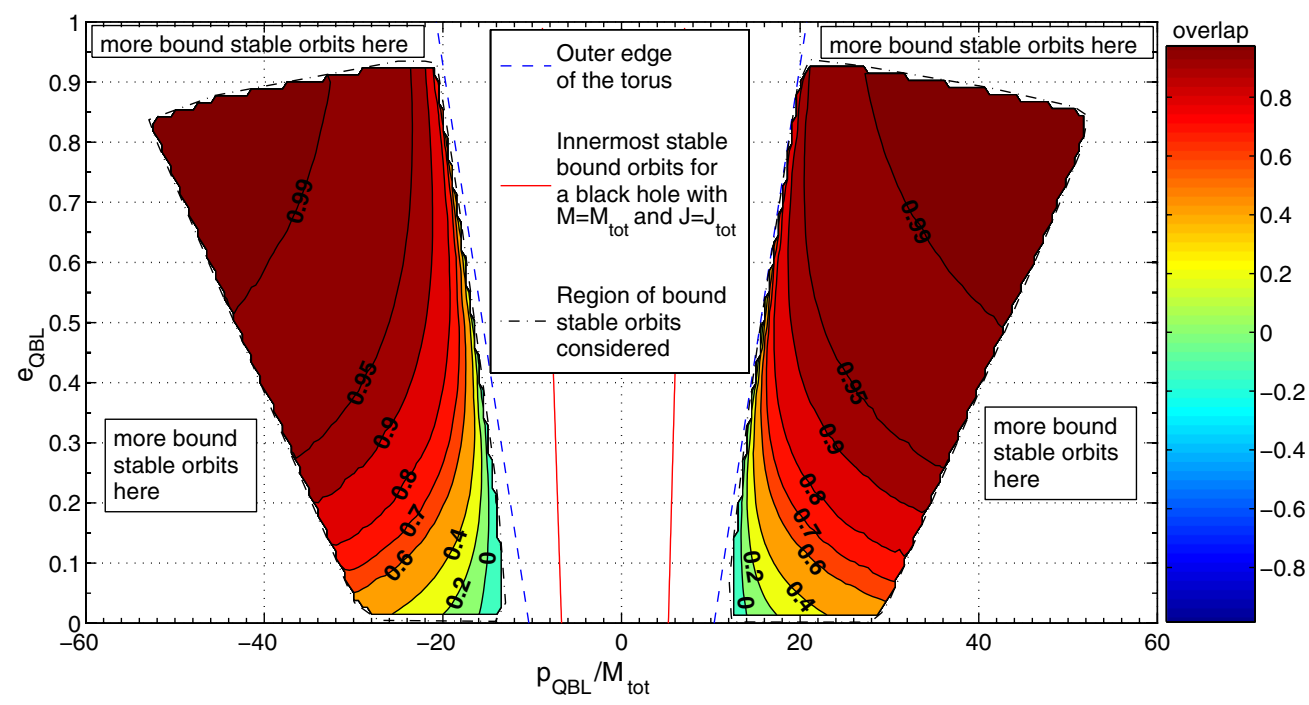

FIG. 4 (color online). The same as in Fig. 3 but for spacetime $B$. Note that in this case the confusion problem is less severe and indeed not present for orbits near the outer edge of the torus (i.e., with $p_{\mathrm{QBL}} / M_{\mathrm{tot}} \lesssim 30$ ) and with eccentricities $e_{\mathrm{QBL}} \lesssim 0.2$.

$\mathcal{O}\left(h_{1}^{\times}, h_{2}^{\times}\right)$for the overlaps plotted in the figures of the next sections is typically smaller than 0.005 and in no case larger than 0.025 .

Finally, we note that the results presented in the next sections refer to a small body with $m=1 M_{\odot}$ and to a sensitivity function for LISA computed using the online generator [59] with its default parameters and, in particular, no white-dwarf noise. As pointed out in Ref. [21], including white-dwarf noise would only lead to a slight increase in the dephasing time.

\section{A. The confusion problem when varying $e$ and $p$}

After excluding a comparison between geodesics (and hence waveforms) that have the same latus rectum and eccentricity in the pure and nonpure Kerr spacetimes because of the very small overlap they produce, we have compared waveforms having the same $r$ - and $\phi$-frequencies as obtained by changing the latus rectum and eccentricity while keeping $M_{\text {Kerr }}$ and $J_{\text {Kerr }}$ fixed [cf. Eqs. (36) and (37)]. More specifically, we already mention that the values of $\delta p / p_{\mathrm{QBL}}$ obtained in the regions of the space of parameters $\left(p_{\mathrm{QBL}}, e_{\mathrm{QBL}}\right)$ where the overlap between these waveforms is high $(\mathcal{O}>0.95)$ are $\left|\delta p / p_{\mathrm{QBL}}\right| \lesssim 0.05$ in spacetime $A$ and $\left|\delta p / p_{\mathrm{QBL}}\right| \lesssim 0.16$ in spacetime $B$. Similarly, the values of $\delta e$ obtained in the regions of the space of parameters where $\mathcal{O}>0.95$ are $|\delta e| \lesssim 0.06$ in spacetime $A$ and $|\delta e| \lesssim 0.07$ in spacetime $B$.

We have computed the overlap between $h_{\mathrm{BH}+\text { Torus }}\left(p_{\mathrm{QBL}}, e_{\mathrm{QBL}}\right)$ and $h_{\mathrm{Kerr}}\left(p_{\mathrm{BL}}=p_{\mathrm{QBL}}+\delta p, e_{\mathrm{BL}}=\right.$ $\left.e_{\mathrm{QBL}}+\delta e, M_{\mathrm{Kerr}}, J_{\mathrm{Kerr}}\right)$, and summarize the results of this comparison for a large set of orbits in Figs. 3-5. In particular, Fig. 3 shows the color-coded overlap between waveforms produced in spacetime $A$ by external orbits in
$\mathrm{a}\left(p_{\mathrm{QBL}}, e_{\mathrm{QBL}}\right)$ plane, with positive values of $p_{\mathrm{QBL}}$ referring to prograde orbits, and negative ones to retrograde orbits. Note that no internal orbits were found in spacetime $A$ and this is due to the fact that in this case the torus is too close to the black hole for bound stable orbits to exist in region I of Fig. 1 without plunging into the black hole. The different lines in Fig. 3 mark the margins of the different regions of interest in the $\left(p_{\mathrm{QBL}}, e_{\mathrm{QBL}}\right)$ plane, with the dashed line representing the outer "edge of the torus," that is the set of points such that $p_{\mathrm{QBL}} /\left(1+e_{\mathrm{QBL}}\right)=r_{\mathrm{out}, \mathrm{QBL}}$. Similarly, the solid line represents the innermost stable bound orbits (this line is also referred to as the "separatrix" in Ref. [60]) for a Kerr spacetime with mass $M_{\text {Kerr }}=M_{\text {tot }}$ and spin $J_{\text {Kerr }}=J_{\text {tot }}$. Finally, the dot-dashed line limits the regions of the $\left(p_{\mathrm{QBL}}, e_{\mathrm{QBL}}\right)$ plane where bound stable orbits have been studied.

We underline that these are not the only regions in which bound stable orbits exist, but they rather represent the regions we have investigated because of their being more directly related to LISA observations. In practice, we exploit the fact that there is a one-to-one correspondence between the latus rectum $p_{\mathrm{QBL}}$ and the eccentricity $e_{\mathrm{QBL}}$ of bound stable orbits and their QI radius and tangential velocity (measured by a ZAMO) at periastron, $r_{p}$ and $v_{\phi}$. We therefore choose the initial radial QI position $r_{0}$ of the small body randomly in a limited range and vary its initial tangential velocity $v_{\phi}$ with small steps in the range of the velocities leading to energies per unit mass $\tilde{\boldsymbol{\epsilon}}<1 .{ }^{8}$ After

\footnotetext{
${ }^{8}$ We note that in both spacetime $A$ and $B$ all the equatorial bound stable orbits not crossing the torus have $\tilde{\epsilon}<1$ [this can be verified by computing the values of $\tilde{\epsilon}$ for which the potential $V(r, \tilde{\epsilon}, \tilde{\ell})$ in Eq. (33) is positive]. However, bound stable orbits which cross the torus and have $\tilde{\epsilon}>1$ are present in both spacetimes.
} 


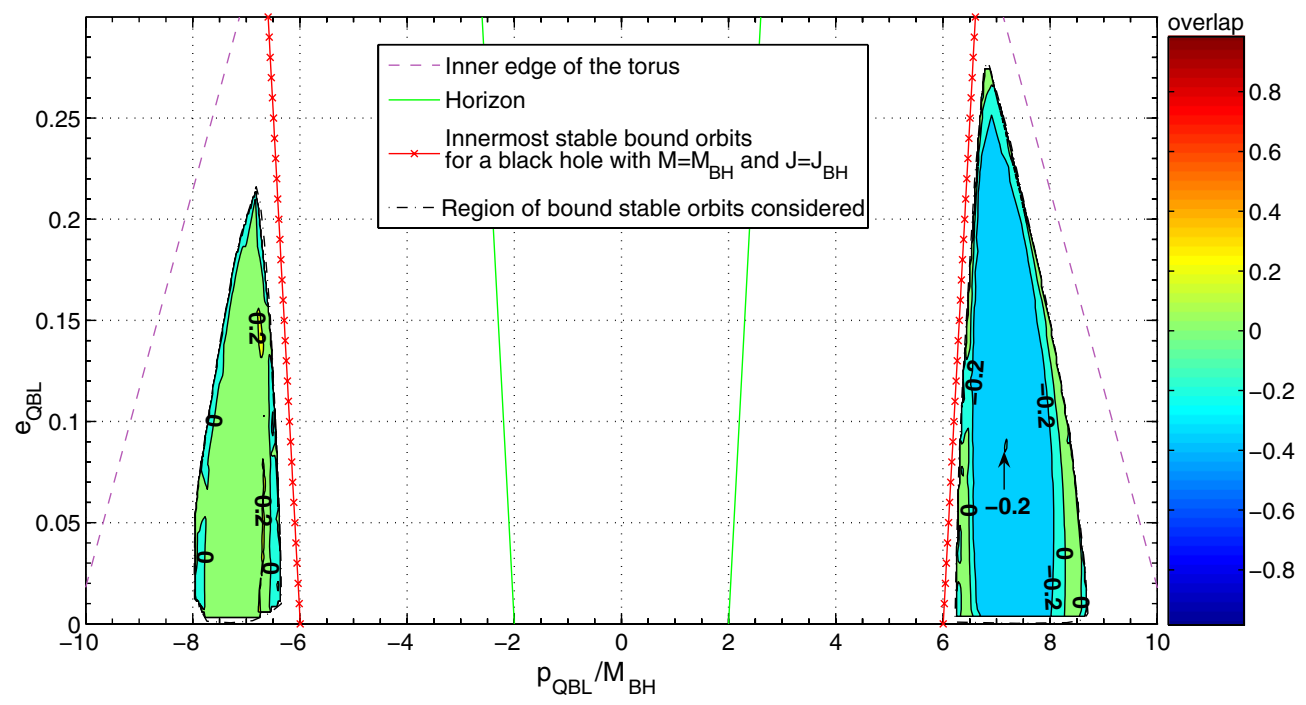

FIG. 5 (color online). The same as in Fig. 4 but for internal orbits, with the solid line marking those orbits whose periastron lies on the event horizon, the dashed line representing the inner "edge of the torus," and finally the crossed-solid line marking the innermost bound stable orbits in a Kerr spacetime with mass and $\operatorname{spin} M_{\mathrm{Kerr}}=M_{\mathrm{BH}}$ and $J_{\mathrm{Kerr}}=J_{\mathrm{BH}}$. Again, the dot-dashed line limits the regions of the $\left(p_{\mathrm{QBL}}, e_{\mathrm{QBL}}\right)$ plane where bound stable orbits have been studied, but in contrast to the case of external orbits, these regions correspond to practically all the bound stable orbits not crossing the torus. Note that in this case the confusion problem is absent, with $\mathcal{O} \lesssim 0.2$.

integrating the geodesic equations (Eqs. (23)-(25) with $\theta=\pi / 2$ ) over and beyond the dephasing time, if the orbit does not intersect the torus and if $r_{0}$ actually corresponds to the periastron (and not to the apoastron) we extract the latus rectum and eccentricity so as to populate the $\left(p_{\mathrm{QBL}}, e_{\mathrm{QBL}}\right)$ plane and compute the overlaps with pure Kerr waveforms (the orbits in the Kerr spacetime are chosen to start at their periastron as well). Overall, a large number of bound stable orbits (i.e. $\gtrsim 2250$ ) have been integrated for each of the figures shown in this paper. Notice that the requirement that $r_{0}$ correspond to the periastron is important because, as far as the overlaps are concerned, orbits having the same latus rectum and eccentricity but different initial positions are not equivalent. We recall in fact that the overlaps are computed by putting a cutoff at the dephasing time and if the initial positions are different, the portions of the orbits contributing to the overlap are different.

Overall, because the waveforms agree very well with an overlap $\mathcal{O}>0.95$ for most of the orbits we have considered, the results in Fig. 3 clearly show that a confusion problem similar to the one presented in Ref. [21] is indeed possible in this spacetime for observational time scales below or comparable to the dephasing time. As indicated by the color coding, the overlap has a drastic reduction only in a limited region of the space of parameters and, in particular, for orbits with small eccentricity and close to the innermost bound stable orbits. This is not surprising as in these regions the local modifications of the spacetime due to the presence of the torus are the largest and have a more marked impact on the waveforms. Interestingly, pro- grade orbits produce overlaps that are smaller than those produced by retrograde orbits with comparable values of $p_{\mathrm{QBL}}$ and $e_{\mathrm{QBL}}$, and appear therefore to be better tracers of this spacetime.

It is important to underline that the presence of an albeit small region of the space of parameters in which the overlap is small, and hence the dangers of a confusion problem decreased, represents an important difference compared to the results presented in Ref. [21]. We recall that spacetime $A$ has a rather small quadrupole parameter $\epsilon$ (cf. Table I), comparable with those used in Ref. [21]. Yet, the small overlaps near the innermost bound stable orbits indicate that taking into account the higher-order multipoles neglected in the metric (21) can lead to significant differences even far away from the black hole if a matter source is present.

Figure 4 summarizes a set of results similar to those presented in Fig. 3 but for spacetime $B$. More specifically, it reports the color-coded overlap between waveforms produced in spacetime $B$ by external orbits and waveforms produced in a Kerr spacetime with mass $M_{\text {Kerr }}=M_{\text {tot }}$ and spin $J_{\text {Kerr }}=J_{\text {tot }}$. Here again, all of the orbits have the same orbital frequencies as obtained by adjusting $\delta p$ and $\delta e$. It should be noted that in this case the confusion problem is less severe and indeed essentially absent for orbits near the outer edge of the torus (i.e., with $p_{\mathrm{QBL}} / M_{\text {tot }} \lesssim 30$ ) and with eccentricities $e_{\mathrm{QBL}} \lesssim 0.2$. Finally, we report in Fig. 5 again results for spacetime $B$ but this time for internal orbits. We recall, in fact, that in this case the torus is farther away from the black hole and thus bound stable orbits can be found in region I of Fig. 1. As in the previous figures, the 
dot-dashed line limits the regions of the plane $\left(p_{\mathrm{QBL}}, e_{\mathrm{QBL}}\right)$ where bound stable orbits have been studied, but in contrast to the case of external orbits these regions correspond to practically all the bound stable orbits not crossing the torus. On the other hand, the solid line marks those orbits whose periastron lies on the event horizon, the dashed line those orbits whose apoastron lies on the inner edge of the torus, and finally the crossed-solid line indicates the innermost bound stable orbits in a Kerr spacetime with mass and spin $M_{\mathrm{Kerr}}=M_{\mathrm{BH}}$ and $J_{\mathrm{Kerr}}=J_{\mathrm{BH}}$. Clearly, no confusion problem is present for these orbits, because the overlap is always very small and never larger than $\simeq 0.2$.

In summary, the overlap computed in the two spacetimes $A$ and $B$ containing a black hole and a torus by varying the latus rectum and the eccentricity reveals that there are regions in which the nonpure Kerr spacetimes can be "confused" with Kerr spacetimes that are equivalent to them at the sensitivity of LISA. Clearly, this risk is concrete only for time scales over which radiation-reaction effects are negligible and it is not present for external orbits very close to the torus or for the orbits between the torus and the black hole, if they exist.

\section{B. The confusion problem when varying $M$ and $J$}

Next, we consider the overlap obtained by comparing orbits having the same $r$ - and $\phi$-frequencies, which was achieved by changing the mass and spin of the Kerr black hole while keeping the latus rectum and eccentricity fixed in either (Q)BL or QI coordinates [cf. Eqs. (38) and (39) and (40) and (41)]. Doing this corresponds to considering a hypothetical scenario in which it would be possible to measure, through independent astronomical observations, the latus rectum and eccentricity of the small body orbiting around the massive central black hole. In practice, and using the same compact notation introduced above, we have compared waveforms of the type $h_{\mathrm{BH}+\text { Torus }}\left(p_{\mathrm{QBL}}, e_{\mathrm{QBL}}\right) \quad$ with $\quad h_{\mathrm{Kerr}}\left(p_{\mathrm{BL}}=p_{\mathrm{QBL}}, e_{\mathrm{BL}}=\right.$ $\left.e_{\mathrm{QBL}}, M_{\mathrm{Kerr}}+\delta M, J_{\mathrm{Kerr}}+\delta J\right)$ [i.e., latus rectum and eccentricity fixed in $(\mathrm{Q}) \mathrm{BL}$ coordinates, $\delta M$ and $\delta J$ solutions to Eqs. (38) and (39)], and $h_{\mathrm{BH}+\mathrm{Torus}}\left(p_{\mathrm{QI}}, e_{\mathrm{QI}}\right)$ to $h_{\mathrm{Kerr}}\left(p_{\mathrm{QI}}, e_{\mathrm{QI}}, M_{\mathrm{Kerr}}+\delta M, J_{\mathrm{Kerr}}+\delta J\right)$ [i.e., latus rectum and eccentricity fixed in QI coordinates, $\delta M$ and $\delta J$ solutions to Eqs. (40) and (41)].

While formally distinct, these two approaches yield essentially the same results quite irrespective of whether the latus rectum and eccentricity are held fixed in $(\mathrm{Q}) \mathrm{BL}$ or in QI coordinates. Because of this, hereafter we will discuss only the results obtained when fixing $p_{(\mathrm{Q}) \mathrm{BL}}$ and $e_{(\mathrm{Q}) \mathrm{BL}}$.

Figure 6, in particular, shows the overlap between waveforms produced in spacetime $A$ by external orbits and waveforms produced in a Kerr spacetime with mass $M_{\text {Kerr }}+\delta M=M_{\text {tot }}+\delta M$ and spin $J_{\text {Kerr }}+\delta J=$ $J_{\text {tot }}+\delta J$ by orbits with the same $p_{(\mathrm{Q}) \mathrm{BL}}$ and $e_{(\mathrm{Q}) \mathrm{BL}}$ and the same orbital frequencies. As in Fig. 3, the different lines mark the margins of the relevant regions of the $\left(p_{\mathrm{QBL}}, e_{\mathrm{QBL}}\right)$ plane, with the dashed line representing the outer "edge of the torus," the solid line representing the innermost stable bound orbits for a Kerr spacetime with mass $M_{\text {Kerr }}=M_{\text {tot }}$ and spin $J_{\text {Kerr }}=J_{\text {tot }}$, and the dot-

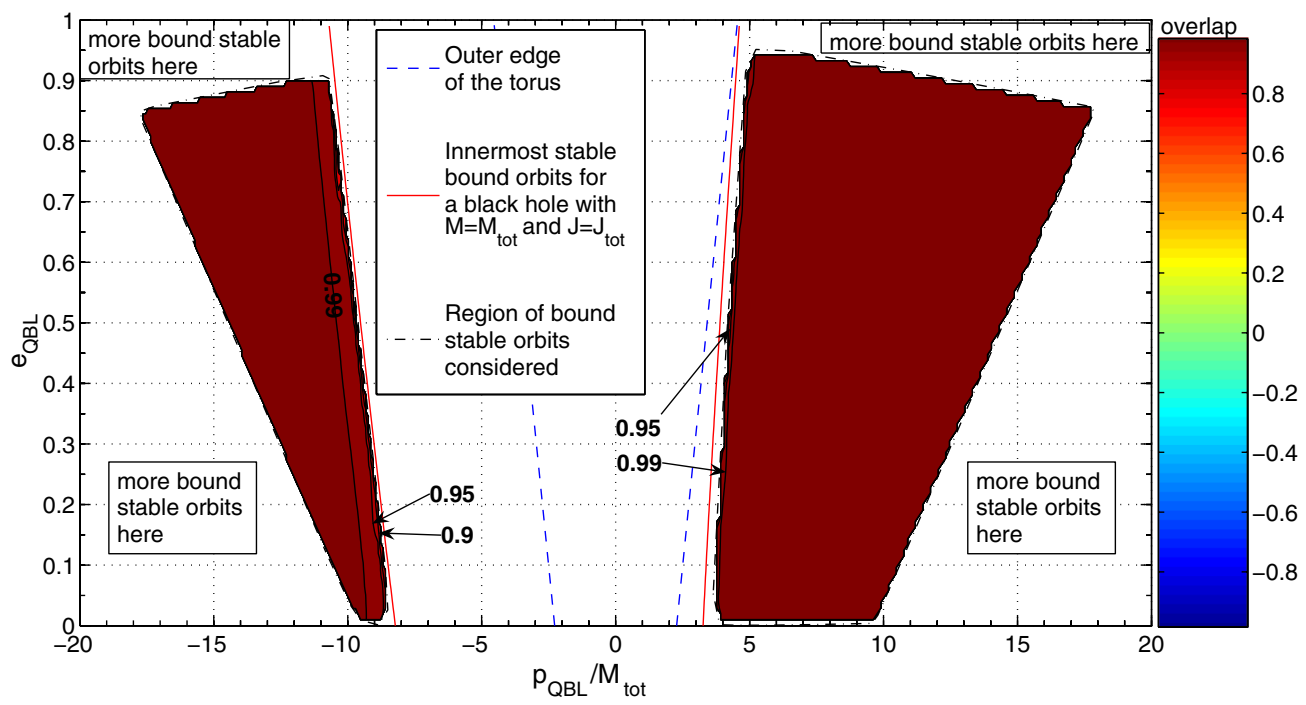

FIG. 6 (color online). Overlap between waveforms produced in spacetime $A$ by external orbits and waveforms produced in a Kerr spacetime with mass $M_{\text {Kerr }}+\delta M=M_{\text {tot }}+\delta M$ and spin $J_{\text {Kerr }}+\delta J=J_{\text {tot }}+\delta J$ by orbits with the same latus rectum and eccentricity [in (Q)BL coordinates] and the same $r$ - and $\phi$-frequencies. Here too, the dashed line represents the outer "edge of the torus," the solid line the innermost stable bound orbits for a Kerr spacetime with mass $M_{\text {Kerr }}=M_{\text {tot }}$ and spin $J_{\text {Kerr }}=J_{\text {tot }}$, and the dot-dashed line limits the regions of the $\left(p_{\mathrm{QBL}}, e_{\mathrm{QBL}}\right)$ plane where bound stable orbits have been studied. An overlap $\mathcal{O}>0.95$ is present in all of the relevant regions of the $\left(p_{\mathrm{QBL}}, e_{\mathrm{QBL}}\right)$ plane. 


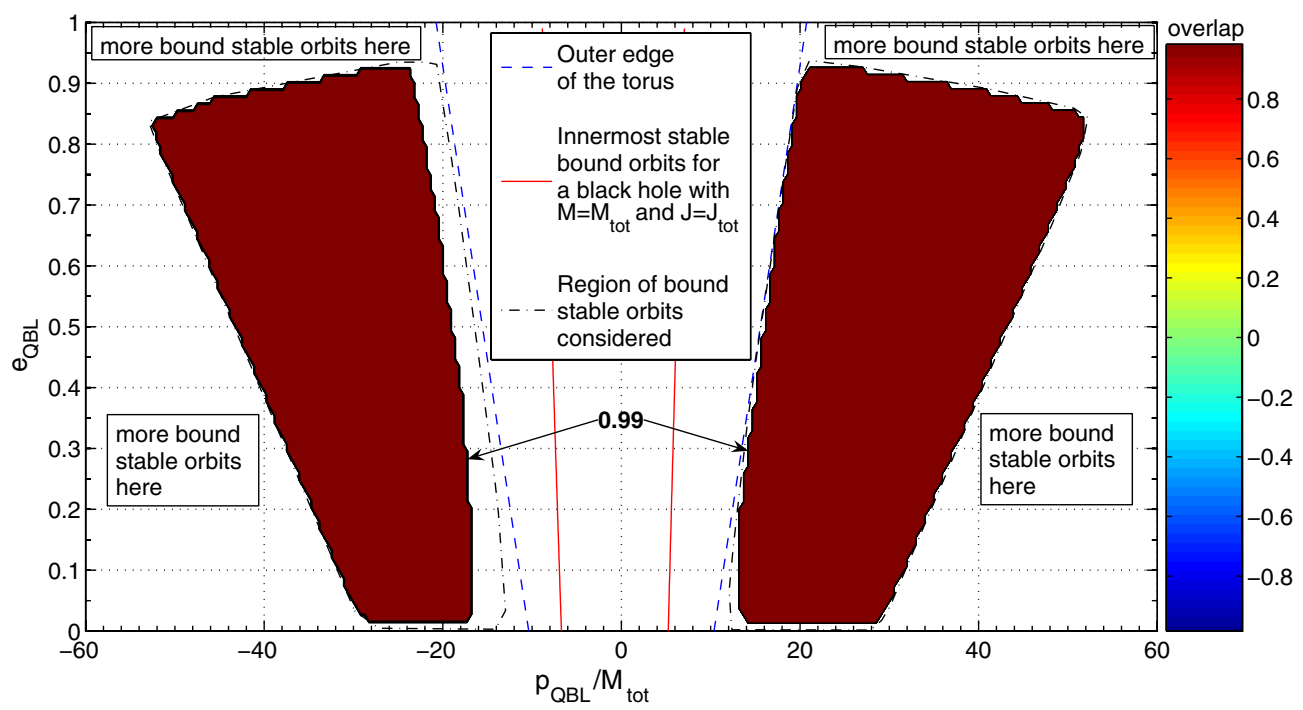

FIG. 7 (color online). The same as in Fig. 6 but for spacetime $B$. Note that also in this case the overlap is very high $(\mathcal{O}>0.99)$ in almost all of the relevant regions of the $\left(p_{\mathrm{QBL}}, e_{\mathrm{QBL}}\right)$ plane, with the exception of a very small set of orbits very close to the torus, for which Eqs. (38) and (39) have no solutions (these orbits correspond to the blank regions inside the dot-dashed line).

dashed line limiting the regions of the $\left(p_{\mathrm{QBL}}, e_{\mathrm{QBL}}\right)$ plane where bound stable orbits have been studied. Note the very close match between the two waveforms, with an overlap $\mathcal{O}>0.95$ in essentially all of the relevant regions of the $\left(p_{\mathrm{QBL}}, e_{\mathrm{QBL}}\right)$ plane. This is a clear indication that a confusion problem is present for LISA measurements over a time scale below or comparable to the dephasing time.
Figures 7 and 8 provide complementary information for spacetime $B$, with the first one referring to external orbits and the second one to internal ones (the meaning of the lines appearing in this figures is the same as in Figs. 4 and 5). In both cases it is apparent that the overlap is always very large. The only exceptions are the internal orbits with the largest allowed eccentricities, for which the overlap

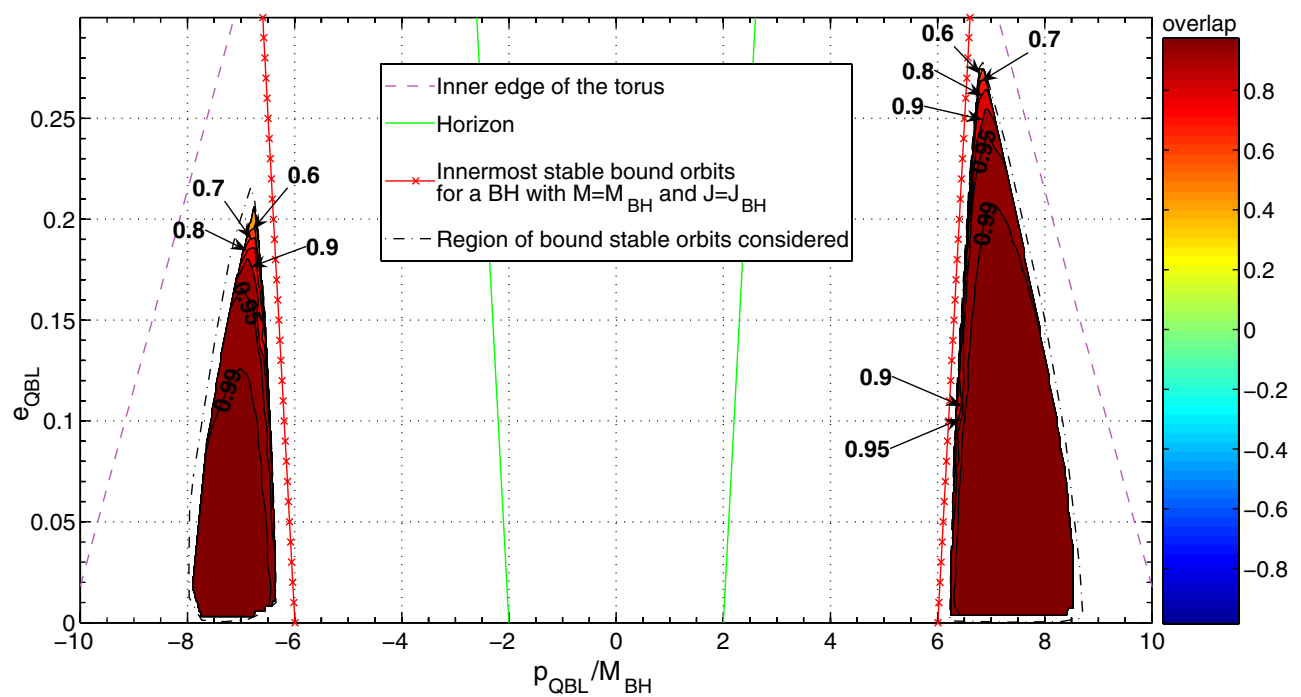

FIG. 8 (color online). The same as in Fig. 7 but for internal orbits, with the solid line marking those orbits whose periastron lies on the event horizon, the dashed line representing the inner "edge of the torus," and finally the crossed-solid line marking the innermost bound stable orbits in a Kerr spacetime with mass and spin $M_{\mathrm{Kerr}}=M_{\mathrm{BH}}$ and $J_{\mathrm{Kerr}}=J_{\mathrm{BH}}$. Again, the dot-dashed line limits the regions of the $\left(p_{\mathrm{QBL}}, e_{\mathrm{QBL}}\right)$ plane where bound stable orbits have been studied, but in contrast to the case of external orbits these regions correspond to practically all the bound stable orbits not crossing the torus. Note that in this case the confusion problem is present in most of the relevant regions of the $\left(p_{\mathrm{QBL}}, e_{\mathrm{QBL}}\right)$ plane, becoming slightly less severe only for the largest allowed eccentricities and for a very small set of orbits, very close to the torus, for which Eqs. (38) and (39) have no solutions (these orbits correspond to the blank regions inside the dot-dashed line). 
decreases slightly, and a very small set of orbits very close to the torus, for which Eqs. (38) and (39) have no solutions (these orbits correspond to the blank regions inside the dotdashed line in Figs. 7 and 8).

In summary, the overlap computed in the two spacetimes by varying the mass and spin of the black hole reveals that a LISA observation carried out over a time scale below or comparable to the dephasing time would not allow an observer to distinguish between a Kerr and a nonpure Kerr spacetime, even in the case in which the orbital parameters of the small body, such as the latus rectum and the eccentricity, were known through astronomical observations.

A simple explanation of why the overlap is always so large when calculated by varying the mass and spin of the Kerr black hole is already illustrated in Fig. 2. This shows that the waveform obtained in this way captures not only the proper orbital frequencies, but also the overall "form" of the signal, which is most sensitive to the values of the latus rectum and of the eccentricity of the orbit (cf. the solid line and the circles in Fig. 2).

The difficulty of distinguishing a Kerr spacetime from a nonpure Kerr one can also be expressed in terms of the mass $M_{\text {Kerr }}+\delta M$ and spin $J_{\text {Kerr }}+\delta J$ that would be measured by an observer analyzing a gravitational wave from a black hole-torus system with pure Kerr templates. The corrections $\delta M$ and $\delta J$ are those appearing in Eqs. (38) and (39) and have been computed to determine the overlaps presented in this section. If they are small and slowly varying, it is hard to imagine a way in which the nonpure Kerr spacetime could be distinguished from a pure Kerr one, even with the help of additional astronomical observations. Conversely, if these corrections are large or rapidly varying it is possible that additional astronomical informa- tion on the system or an analysis of snapshots of the waveform taken at different times could be used to determine that the source is not an isolated Kerr black hole and therefore lessen the confusion problem we find in our analysis.

A synthesis of these corrections for the determination of the mass of the black hole in the case of spacetime $B$ is presented in Figs. 9 and 10, with the first one showing the relative error $\delta M / M_{\text {Kerr }}=\delta M / M_{\text {tot }}$ in the regions of the $\left(p_{\mathrm{QBL}}, e_{\mathrm{QBL}}\right)$ plane where the overlap plotted for external orbits is above 0.95 , and the second one showing the corresponding quantity $\left(\delta M / M_{\text {Kerr }}=\delta M / M_{\mathrm{BH}}\right)$ for internal orbits.

Clearly, the corrections are very small and slowly varying in almost all of the relevant space of parameters for external orbits, meaning that an observer could not detect the presence of the torus using only these orbits. On the other hand, an observer could measure rather accurately the total mass of the system. Note, in particular, that the correction $\delta M / M_{\text {tot }}$ goes to zero far from the system, as one would expect.

This situation is only slightly different for internal orbits, for which the correction increases to some percent: using internal orbits an observer could measure quite accurately the mass of the central black hole. Note therefore that a combination of observations of internal orbits (giving an estimate for $M_{\mathrm{BH}}$ ) and external orbits (giving an estimate for $M_{\mathrm{tot}}$ ) could hint at the presence of a torus around the central black hole.

Similar behavior has also been found for spacetime $A$. Because no internal bound stable orbits are present, an observer could not measure the individual masses of the black hole and the torus, whereas he could measure accurately the total mass of the system. In fact, the corrections

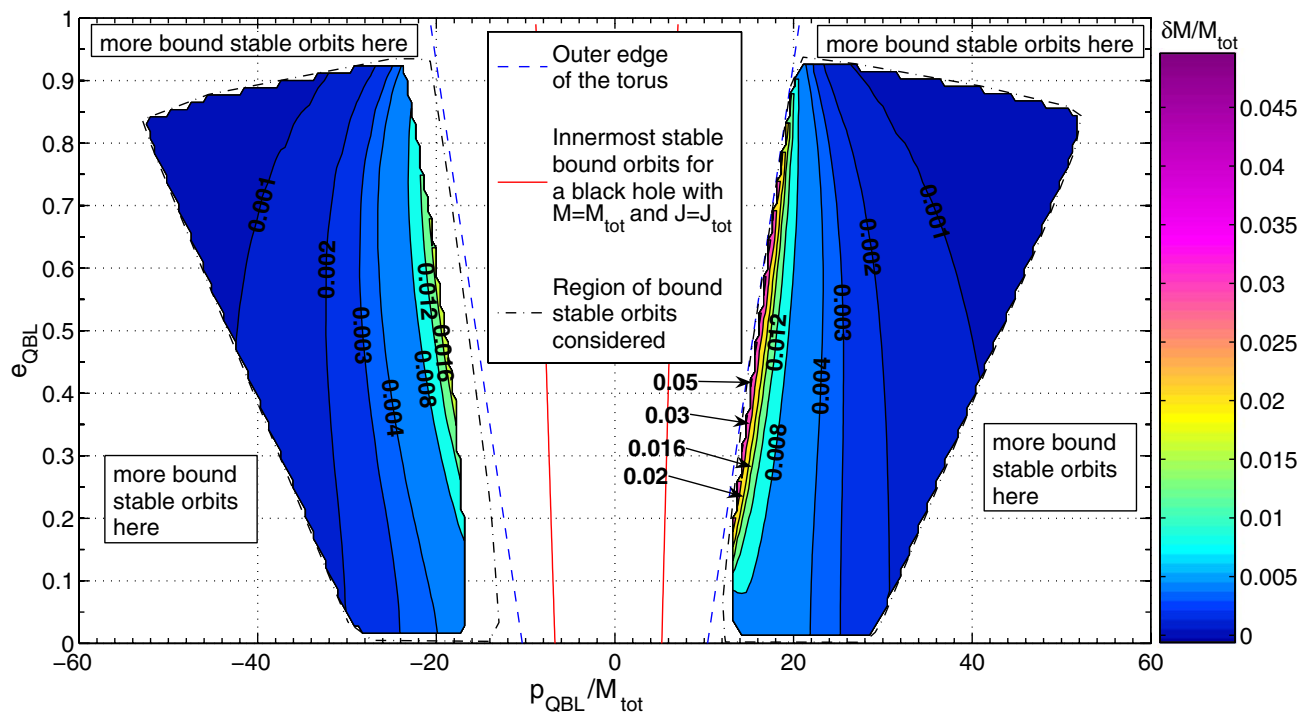

FIG. 9 (color online). Relative mass correction $\delta M / M_{\mathrm{Kerr}}=\delta M / M_{\mathrm{tot}}$ in the regions of the $\left(p_{\mathrm{QBL}}, e_{\mathrm{QBL}}\right)$ plane where the overlap plotted in Fig. 7 is above 0.95. Note that far from the system $\delta M / M_{\text {tot }}$ approaches zero, as one would expect. 


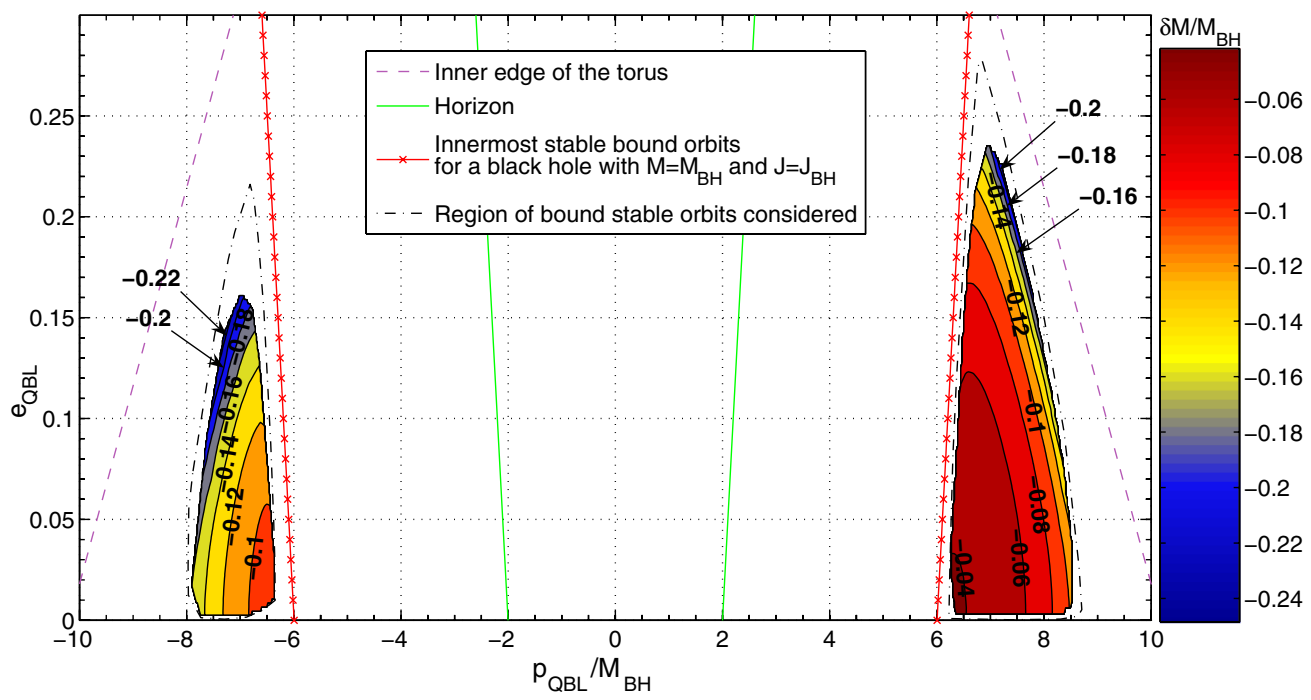

FIG. 10 (color online). The same as in Fig. 9 but for internal orbits. In this case the deviations are computed as $\delta M / M_{\mathrm{Kerr}}=$ $\delta M / M_{\mathrm{BH}}$ in the regions of the $\left(p_{\mathrm{QBL}}, e_{\mathrm{QBL}}\right)$ plane where the overlap plotted in Fig. 8 is above 0.95 .

are always very small with $\left|\delta M / M_{\text {Kerr }}\right|=\left|\delta M / M_{\text {tot }}\right| \lesssim$ 0.02 ; again, the correction $\delta M / M_{\text {tot }}$ goes to zero far from the system, as one would expect. Note that due to the absence of internal orbits in this spacetime and to the smallness and slow variations of $\delta M / M_{\text {tot }}$ it is extremely difficult to distinguish spacetime $A$ from a pure Kerr spacetime.

Information complementary to the one given by the mass correction $\delta M$ is offered by the spin correction $\delta J$. In particular, for spacetime $A$ the correction $\delta a$ defined by $\delta a \equiv\left(J_{\mathrm{Kerr}}+\delta J\right) /\left(M_{\mathrm{Kerr}}+\delta M\right)^{2}-a_{\mathrm{Kerr}}$ (with $a_{\mathrm{Kerr}}=$ $\left.J_{\text {Kerr }} / M_{\text {Kerr }}^{2}=0.728\right)$ can be readily calculated to be $\left|\delta a / a_{\text {Kerr }}\right| \lesssim 0.065$, going to zero, as one would expect, far from the system. This means that an observer could accurately measure the total spin of the black hole-torus system although, due to the absence of internal orbits in this system and to the slow variations of $\delta a$, a measurement of the individual spins of the torus and the black hole or even a simple detection of the torus seems unfeasible.

Spacetime $B$ is considered in Figs. 11 and 12, in which we report the quantity $a_{\text {Kerr }}+\delta a \equiv\left(J_{\text {Kerr }}+\right.$ $\delta J) /\left(M_{\text {Kerr }}+\delta M\right)^{2}$ for external (with $a_{\text {Kerr }}=$ $J_{\text {Kerr }} / M_{\text {Kerr }}^{2}=0.224$ ) and internal (with $a_{\text {Kerr }}=$ $J_{\text {Kerr }} / M_{\text {Kerr }}^{2}=-1.74 \times 10^{-3}$ ) orbits, respectively. As can be seen, the corrections $\delta a$ are, in both cases, rather large and rapidly varying: an observer could probably distin-

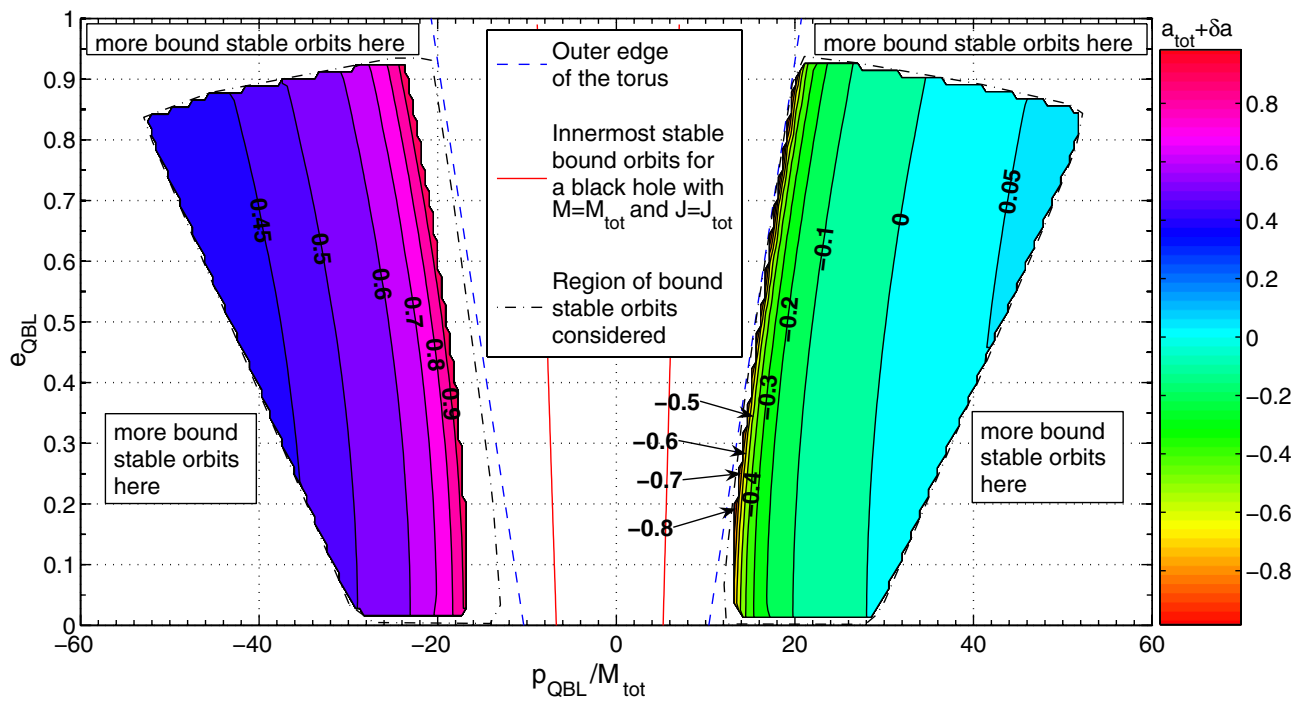

FIG. 11 (color online). Variations of the spin $a_{\mathrm{Kerr}}+\delta a \equiv a_{\mathrm{tot}}+\delta a=\left(J_{\mathrm{tot}}+\delta J\right) /\left(M_{\mathrm{tot}}+\delta M\right)^{2}$ in the regions of the $\left(p_{\mathrm{QBL}}, e_{\mathrm{QBL}}\right)$ plane where the overlap plotted in Fig. 7 is above 0.95 . We recall that for external orbits in spacetime $B$, we have $a_{\text {Kerr }}=a_{\text {tot }}=0.224$ (cf. Table I). 


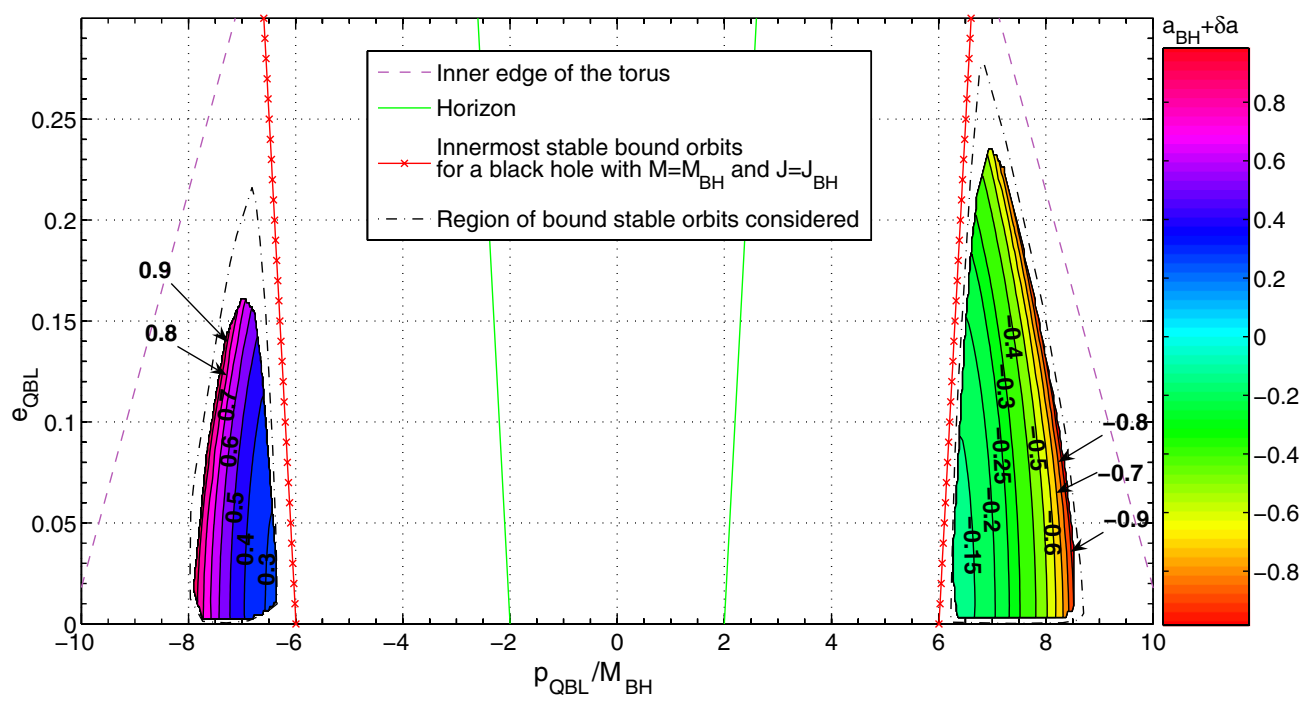

FIG. 12 (color online). The same as in Fig. 11 but for internal orbits. In this case the corrections are computed as $a_{\mathrm{Kerr}}+\delta a=$ $a_{\mathrm{BH}}+\delta a \equiv\left(J_{\mathrm{BH}}+\delta J\right) /\left(M_{\mathrm{BH}}+\delta M\right)^{2}$ in the regions of the $\left(p_{\mathrm{QBL}}, e_{\mathrm{QBL}}\right)$ plane where the overlap plotted in Fig. 8 is above 0.95 . We recall that for internal orbits in spacetime $B$, we have $a_{\mathrm{Kerr}}=a_{\mathrm{BH}}=-1.74 \times 10^{-3}$ (cf. Table I).

guish this spacetime from a pure Kerr one using estimates of the spin obtained by analyzing the waveform at different times, but would have little chance to measure the spin of the central black hole correctly and should consider orbits very far from the system in order to achieve accurate measurements of the total spin. This was to be expected, since spacetime $B$ has a large ratio $J_{\text {Torus }} / J_{\mathrm{BH}}$, which causes the quadrupole parameter $\epsilon$ to be large (cf. Table I).

Before concluding this section, it is worth commenting on how robust and generic these results are. While we believe they represent the first attempt to model consistently the gravitational-wave emission from spacetimes that deviate considerably for Kerr due to the presence of matter, the approach followed here has the obvious limitation of neglecting radiation-reaction effects and thus of considering waveforms only over a dephasing time which is typically of days or weeks. It is therefore possible, if not likely, that considering waveforms over a time scale comparable with LISA's planned lifetime (i.e. 3-5 years) would lower the overlaps computed here and thus reduce the impact of a confusion problem.

As already mentioned, a simple way to include radiation reaction would consist of using the adiabatic approximation and thus considering motion along a geodesic with slowly changing parameters. In particular, approximate (kludge) expressions for the fluxes $\dot{E}, \dot{L}_{z}$, and $\dot{Q}$ in Kerr have been derived using post-Newtonian expansions $[51,52]$, recently corrected using fits to the fluxes computed rigorously with the Teukolsky formalism [53]. Likewise, it may be possible to adopt similar strategies in non-Kerr spacetimes. For instance, Cutler and Barack [25] recently proposed including radiation reaction in quasi-Kerr spacetimes by using post-Newtonian fluxes in which the leadingorder effect of the quadrupole of the spacetime is taken into account, potentially eliminating the confusion problem. Nevertheless, it is still unclear at this stage whether postNewtonian fluxes will be a good approximation for our spacetimes, where the parameters $\epsilon$ and $a$ can be $O(1)$. We recall, indeed, that using post-Newtonian fluxes is not always a good approximation even in Kerr spacetimes and that the most accurate kludge fluxes for Kerr [53] are certainly based on post-Newtonian expansions, but are also corrected using fits to rigorous Teukolsky-based fluxes.

We also note than even with the radiation reaction included, a confusion problem might in principle still be present, at least for equatorial orbits. In fact, requiring the equality of the $r$-and $\phi$-frequencies fixes only two of the four free parameters characterizing the geodesic, $p, e$, $M_{\mathrm{Kerr}}$, and $J_{\mathrm{Kerr}}$, while the remaining two could be used to obtain the equality of the time derivatives of the $r$-and $\phi$-frequencies at the initial time $\left[\dot{\omega}_{r}^{\mathrm{BH}+\text { Torus }}\left(t_{0}\right)=\right.$ $\left.\dot{\omega}_{r}^{\mathrm{Kerr}}\left(t_{0}\right), \dot{\omega}_{\phi}^{\mathrm{BH}+\mathrm{Torus}}\left(t_{0}\right)=\dot{\omega}_{\phi}^{\mathrm{Kerr}}\left(t_{0}\right)\right]$, which could ensure, at least initially, a similar evolution under radiation reaction for the two waveforms.

Besides inclusion of radiation reaction, three other approaches to improve the estimates computed in this paper are also worth considering. The first and most obvious one consists of replacing the kludge waveforms with more rigorous waveforms, solutions of Eqs. (10) and (13), possibly neglecting the fluid perturbations appearing on the right-hand side of Eq. (10) (the latter could be a rather good approximation for orbits far enough from the torus.). Doing this in practice is certainly not trivial since Eqs. (10) have been solved only for a Schwarzschild spacetime [29] so far. The second improvement is simpler and involves considering tori which are not as compact and close to the black hole as the ones studied here, but are instead a better 
approximation of those observed around SMBHs in AGNs. Finally, the third possible improvement involves the extension of the present analysis to nonequatorial orbits. While this is more complicated as one cannot require the strict equality of the orbital frequencies [in contrast to Kerr, Eqs. (23)-(26) indicate that in general the $r$-, $\theta$-, and $\phi$-motions are not periodic in the time coordinate $t$, the motions in the $r$-, $\theta$-, and $\phi$-directions are almost periodic if the torus is not too massive and hence the present analysis can be extended straightforwardly in terms of these almost-periodic motions.

\section{CONCLUSIONS}

EMRIs are expected to be among the most important sources for LISA and, besides mapping accurately the spacetime around SMBHs, they might also shed light on the distribution of matter around them. We have here studied EMRIs and the corresponding gravitational-wave emission in spacetimes that are highly accurate numerical solutions of the Einstein equations and consist of a SMBH and a compact torus with comparable mass and spin. We underline that the tori considered here do not represent a model for the accretion disks in AGNs but, rather, are a phenomenological model for a compact source of matter close to the SMBH. Our goal in this paper has therefore been that of maximizing the impact of this matter on the waveforms, investigating whether gravitational-wave observations will be able to reveal its presence. This hypothetical matter source, even if it exists, may not be detectable otherwise, being too close to the central SMBH and possibly dark.

Using the semirelativistic approach proposed in Ref. [21], we have compared kludge waveforms produced by equatorial orbits in nonpure Kerr spacetimes with waveforms produced by equatorial orbits in Kerr spacetimes having the same mass and spin as the nonpure Kerr spacetimes. Because they are produced by purely geodetic motion, these waveforms are valid only over a rather short "dephasing" time scale. Overall, we find that waveforms produced by orbits having the same latus rectum and eccentricity $p$ and $e$ are considerably different throughout the whole space of parameters $(p, e)$. On the other hand, comparisons of waveforms produced by (equatorial) orbits having the same $r$ - and $\phi$-frequencies, with this condition being achieved by changing the latus rectum and eccentricity of the orbits in the Kerr spacetime, produce overlaps $\mathcal{O}>0.95$ for orbits far from the black hole-torus system, hence pointing out a confusion problem. This overlap decreases rapidly as one considers orbits which are close to the torus, indicating that in the strong-field region no confusion problem is present. Finally, if the equality of the $r$ - and $\phi$-frequencies is obtained by changing the mass and spin of the Kerr spacetime while maintaining fixed the latus rectum and the eccentricity of the orbit, the resulting overlaps are very high, with $\mathcal{O}>0.99$ for essentially all of the orbital parameters $p$ and $e$, indicating a confusion problem that is less severe only for a few orbits very close to the torus.

This confusion problem in the mass and the spin might therefore be more serious than the one involving latus rectum and eccentricity. Stated differently, an observer analyzing below the dephasing time scale a gravitational waveform produced by an EMRI in a black hole-torus system would not be able to distinguish it from one produced in a pure Kerr spacetime. This observer would therefore associate the EMRI to a Kerr SMBH whose mass and spin would however be estimated incorrectly.

While these results represent the first attempt to model consistently the gravitational-wave emission from spacetimes that deviate considerably for Kerr, the approach followed here is based on four approximations, namely: (i) the use of kludge waveforms in place of ones that are consistent solutions of the Einstein equations; (ii) the use of a cutoff at the dephasing time beyond which radiationreaction effects can no longer be ignored; (iii) the restriction to purely equatorial orbits; (iv) the use of tori that are very compact and close to the black hole. Work is now in progress to relax one or more of these approximations, with the expectation that this will lead to a less serious confusion problem.

\section{ACKNOWLEDGMENTS}

It is a pleasure to thank S. Babak, M. Colpi, K. Glampedakis, J. Miller, E. Poisson, T. Sotiriou, and P. Ullio for fruitful and enlightening discussions. E. B. would like to thank T. Bogdanovic for useful comments during the 6th LISA Symposium. E. B. acknowledges support also from the European Network of Theoretical Astroparticle Physics ILIAS/N6 (Contract No. RII3-CT2004-506222). D. P. was supported by the DFG grant SFB/ Transregio 7, "Gravitational Wave Astronomy."

\section{APPENDIX: FROM THE EINSTEIN EQUATIONS TO THE SEMIRELATIVISTIC APPROACH}

Although the main motivation for the semirelativistic approach we use in this paper is the surprising agreement that kludge waveforms show in Kerr with the rigorous waveforms computed using the Teukolsky formalism [50], one can also try to make sense of it using the Einstein equations.

We start by rewriting the Einstein equations in a more convenient form in which we isolate the perturbation as $[58,61]$

$$
\bar{H}^{\mu \nu} \equiv \eta^{\mu \nu}-(-\tilde{g})^{1 / 2} \tilde{g}^{\mu \nu},
$$

where $\eta^{\mu \nu}$ is the Minkowski metric. Since far from the source the spacetime reduces to Minkowski plus a small perturbation, i.e. $\boldsymbol{g}=\boldsymbol{\eta}$, the first-order perturbations there 
coincide with $\overline{\boldsymbol{H}}$, i.e. $\bar{H}^{\mu \nu}=\bar{h}^{\mu \nu}+O(m / M)^{2}$, with $\bar{h}^{\mu \nu}$ being the trace-reversed potentials defined in Eq. (8).

If we now restrict our attention to a region of the spacetime where it is possible to choose the harmonic gauge

$$
\partial_{\beta} \bar{H}^{\alpha \beta}=0
$$

(this is always possible far enough from the source), the full Einstein equations give [61]

$$
\square_{\text {flat }} \bar{H}^{\alpha \beta}=-16 \pi \tau^{\alpha \beta},
$$

where $\square_{\text {flat }} \equiv \eta^{\mu \nu} \partial_{\mu} \partial_{\nu}$ is the flat-spacetime wave operator. The right-hand side is given by the effective stressenergy pseudotensor

$$
\tau^{\alpha \beta}=(-\tilde{g}) \tilde{T}^{\alpha \beta}+(16 \pi)^{-1} \Lambda^{\alpha \beta},
$$

where $\Lambda^{\alpha \beta}$ is given by

$$
\Lambda^{\alpha \beta}=16 \pi(-\tilde{g}) t_{\mathrm{LL}}^{\alpha \beta}+\left(\bar{H}^{\alpha \mu}{ }_{, \nu} \bar{H}^{\beta \nu}{ }_{, \mu}-\bar{H}^{\alpha \beta}{ }_{, \mu \nu} \bar{H}^{\mu \nu}\right),
$$

and $t_{\mathrm{LL}}^{\alpha \beta}$ is the Landau-Lifshitz pseudotensor

$$
\begin{aligned}
16 \pi(-\tilde{g}) t_{\mathrm{LL}}^{\alpha \beta} \equiv & \tilde{g}_{\lambda \mu} \tilde{g}^{\nu \rho} \bar{H}_{, \nu}^{\alpha \lambda} \bar{H}_{, \rho}^{\beta \mu}+\frac{1}{2} \tilde{g}_{\lambda \mu} \tilde{g}^{\alpha \beta} \bar{H}_{, \rho}^{\lambda \nu} \bar{H}_{, \nu}^{\rho \mu} \\
& -2 \tilde{g}_{\mu \nu} \tilde{g}^{\lambda(\alpha} \bar{H}_{, \rho}^{\beta) \nu} \bar{H}_{, \lambda}^{\rho \mu} \\
& +\frac{1}{8}\left(2 \tilde{g}^{\alpha \lambda} \tilde{g}^{\beta \mu}-\tilde{g}^{\alpha \beta} \tilde{g}^{\lambda \mu}\right) \\
& \times\left(2 \tilde{g}_{\nu \rho} \tilde{g}_{\sigma \tau}-\tilde{g}_{\rho \sigma} \tilde{g}_{\nu \tau}\right) \bar{H}_{, \lambda}^{\nu \tau} \bar{H}_{, \mu}^{\rho \sigma} .
\end{aligned}
$$

Because of the gauge condition (A2), the source term of Eq. (A3) satisfies the conservation law

$$
\tau_{, \beta}^{\alpha \beta}=0,
$$

which is equivalent to the equations of motion of the matter

$$
\tilde{\nabla}_{\beta} \tilde{T}^{\alpha \beta}=0 .
$$

Combining then Eqs. (A3) and (A7), in the slow motion approximation one easily gets the usual quadrupole formula (see Ref. [58] for details):

$$
\begin{gathered}
\bar{H}^{i j}(\vec{x}, t)=\frac{2}{r}\left[\frac{d^{2} I^{i j}}{d t^{\prime 2}}\right]_{t^{\prime}=t-r}, \\
I^{i j}\left(t^{\prime}\right)=\int \tau^{00}\left(\vec{x}^{\prime}, t^{\prime}\right) x^{\prime i} x^{\prime j} \mathrm{~d}^{3} x^{\prime},
\end{gathered}
$$

where $r^{2} \equiv \vec{x} \cdot \vec{x}$. Note that one can easily relax the slow motion assumption by including the octupole terms [62] or even all the higher-order multipoles (the formula is due to Press [63]).

Equation (A9) clearly does not allow one to compute $\bar{H}^{i j}$ directly, because its right-hand side depends on $\bar{H}^{\alpha \beta}$ [cf. Eq. (A4)]. The semirelativistic approximation consists indeed in pretending that $\overline{\boldsymbol{H}}$ is "small": making this assumption, one can neglect, in the expression (A4) for the effective stress-energy tensor $\tau^{\alpha \beta}$, the terms quadratic in $\bar{H}^{\alpha \beta}$, and the terms in which $\bar{H}^{\alpha \beta}$ is multiplied by the mass $m$ of the small body. In addition, the semirelativistic approximation also neglects all the terms involving the stressenergy tensor of the fluid: with these assumptions, $\tau^{\alpha \beta}$ can be written as

$$
\begin{gathered}
\tau^{00}(\vec{x}, t)=m \gamma(t) \delta^{(3)}(\vec{x}-\vec{z}(t)), \\
\tau^{0 i}(\vec{x}, t)=m \gamma(t) \dot{z}^{i}(t) \delta^{(3)}(\vec{x}-\vec{z}(t)), \\
\tau^{i j}(\vec{x}, t)=m \gamma(t) \dot{z}^{i}(t) \dot{z}^{j}(t) \delta^{(3)}(\vec{x}-\vec{z}(t)), \\
\gamma \equiv\left(1-\delta_{i j} \dot{z}^{i} \dot{z}^{j}\right)^{-1 / 2},
\end{gathered}
$$

where the dot indicates the derivative with respect to the coordinate time $t$ and the trajectory $z^{i}(t)$ of the small body is obtained by solving the geodesic equations, which are indeed contained in Eq. (A8). Note that Eqs. (A11)-(A13) represent the stress-energy tensor of a small body moving along the trajectory $z^{i}(t)$ in a Minkowski spacetime, which constitutes exactly the assumption on which kludge waveforms are based. In particular, the quadrupole moment (A10) reduces, in the slow motion approximation, to its textbook version $I^{i j}(t)=m z^{i}(t) z^{j}(t)$, while analogous simplifications happen for the octupole and Press formulas (see Ref. [50] for details).

Having calculated $\bar{H}^{i j} \approx \bar{h}^{i j}$, it is then a trivial task to project out the gauge invariant transverse traceless perturbations $h_{+}$and $h_{\times}$at infinity (see for instance Refs. [50,58] for details).
[1] http://lisa.nasa.gov/; http://sci.esa.int/home/lisa/.

[2] J. Kormendy and D. Richstone, Annu. Rev. Astron. Astrophys. 33, 581 (1995).

[3] J. R. Gair, L. Barack, T. Creighton, C. Cutler, S. L. Larson, E. Phinney, and M. Vallisneri, Classical Quantum Gravity 21, S1595 (2004).
[4] P. O. Mazur and E. Mottola, Proc. Natl. Acad. Sci. U.S.A. 101, 9545 (2004).

[5] D. F. Torres, S. Capozziello, and G. Lambiase, Phys. Rev. D 62, 104012 (2000).

[6] F. Munyaneza, D. Tsiklauri, and R. D. Viollier, Astrophys. J. Lett. 509, L105 (1998); R. Schodel et al., Nature 
(London) 419, 694 (2002).

[7] A. A. Svidzinsky, astro-ph/0607179.

[8] A. E. Broderick and R. Narayan, Astrophys. J. Lett. 638, L21 (2006).

[9] R. Antonucci, Annu. Rev. Astron. Astrophys. 31, 473 (1993); C. M. Urry and P. Padovani, Publ. Astron. Soc. Pac. 107, 803 (1995).

[10] J.-M. Huré, Astron. Astrophys. 395, L21 (2002).

[11] R. Genzel et al., Astrophys. J. 594, 812 (2003).

[12] J. F. Navarro, C. S. Frenk, and S. D. M. White, Astrophys. J. 462, 563 (1996); 490, 493 (1997); J. F. Navarro et al., Mon. Not. R. Astron. Soc. 349, 1039 (2004).

[13] O. Y. Gnedin and J.R. Primack, Phys. Rev. Lett. 93, 061302 (2004).

[14] P. Gondolo and J. Silk, Phys. Rev. Lett. 83, 1719 (1999).

[15] P. Ullio, H. Zhao, and M. Kamionkowski, Phys. Rev. D 64, 043504 (2001).

[16] P. Salucci, Mon. Not. R. Astron. Soc. 320, L1 (2001).

[17] P. Sikivie, Phys. Rev. D 60, 063501 (1999); A. Natarajan and P. Sikivie, Phys. Rev. D 73, 023510 (2006).

[18] F. D. Ryan, Phys. Rev. D 52, 5707 (1995).

[19] F. D. Ryan, Phys. Rev. D 56, 1845 (1997).

[20] F. D. Ryan, Phys. Rev. D 56, 7732 (1997).

[21] K. Glampedakis and S. Babak, Classical Quantum Gravity 23, 4167 (2006).

[22] M. Kesden, J. Gair, and M. Kamionkowski, Phys. Rev. D 71, 044015 (2005).

[23] N. A. Collins and S. A. Hughes, Phys. Rev. D 69, 124022 (2004).

[24] M. Ansorg and D. Petroff, Phys. Rev. D 72, 024019 (2005).

[25] L. Barack and C. Cutler, Phys. Rev. D 75, 042003 (2007).

[26] Y. Mino, M. Sasaki, and T. Tanaka, Phys. Rev. D 55, 3457 (1997).

[27] E. Poisson, Living Rev. Relativity 7, 6 (2004).

[28] T.C. Quinn and R.M. Wald, Phys. Rev. D 56, 3381 (1997).

[29] L. Barack and C. O. Lousto, Phys. Rev. D 72, 104026 (2005).

[30] E. Poisson, gr-qc/0410127.

[31] Y. Mino, Phys. Rev. D 67, 084027 (2003).

[32] A. Pound, E. Poisson, and B. G. Nickel, Phys. Rev. D 72, 124001 (2005).

[33] D. V. Gal'tsov, J. Phys. A 15, 3737 (1982).

[34] N. Sago, T. Tanaka, W. Hikida, and H. Nakano, Prog. Theor. Phys. 114, 509 (2005); N. Sago, T. Tanaka, W. Hikida, K. Ganz, and H. Nakano, Prog. Theor. Phys. 115, 873 (2006).

[35] M. J. Pfenning and E. Poisson, Phys. Rev. D 65, 084001 (2002); D. W. Sciama, P. C. Waylen, and R.C. Gilman, Phys. Rev. 187, 1762 (1969).

[36] S. Drasco, Classical Quantum Gravity 23, S769 (2006).
[37] E. Rosenthal, Phys. Rev. D 73, 044034 (2006).

[38] S. Drasco and S.A. Hughes, Phys. Rev. D 73, 024027 (2006).

[39] S. A. Teukolsky, Astrophys. J. 185, 635 (1973); M. Sasaki and T. Nakamura, Prog. Theor. Phys. 67, 1788 (1982).

[40] R. Geroch, J. Math. Phys. (N.Y.) 11, 2580 (1970); R. Hansen, J. Math. Phys. (N.Y.) 15, 46 (1974); G. Fodor, C. Hoenselaers, and Z. Perjés, J. Math. Phys. (N.Y.) 30, 2252 (1989).

[41] P. Kordas, Classical Quantum Gravity 12, 2037 (1995).

[42] R. Meinel and G. Neugebauer, Classical Quantum Gravity 12, 2045 (1995).

[43] J. B. Hartle, Astrophys. J. 150, 1005 (1967); J. B. Hartle and K. S. Thorne, Astrophys. J. 153, 807 (1968).

[44] A. Komar, Phys. Rev. 113, 934 (1959).

[45] J. M. Bardeen, in Black Holes, Les astres Occlus, edited by C. DeWitt and B.S. DeWitt (Gordon and Breach, New York, 1973).

[46] R. Arnowitt, S. Deser, and C. W. Misner, in Gravitation: An Introduction to Current Research, edited by L. Witten (Wiley, New York, 1962).

[47] D. Christodoulou, Phys. Rev. Lett. 25, 1596 (1970).

[48] R. Ruffini and M. Sasaki, Prog. Theor. Phys. 66, 1627 (1981).

[49] K. Glampedakis, Classical Quantum Gravity 22, S605 (2005).

[50] S. Babak, H. Fang, J. R. Gair, K. Glampedakis, and S. A. Hughes, Phys. Rev. D 75, 024005 (2007).

[51] F. D. Ryan, Phys. Rev. D 52, R3159 (1995); 53, 3064 (1996).

[52] K. Glampedakis, S. A. Hughes, and D. Kennefick, Phys. Rev. D 66, 064005 (2002).

[53] J. R. Gair and K. Glampedakis, Phys. Rev. D 73, 064037 (2006).

[54] C. Cutler and É.E. Flanagan, Phys. Rev. D 49, 2658 (1994).

[55] R. Balasubramanian, B.S. Sathyaprakash, and S. V. Dhurandhar, Phys. Rev. D 53, 3033 (1996).

[56] W. Schmidt, Classical Quantum Gravity 19, 2743 (2002).

[57] G. Cook, Living Rev. Relativity 3, 5 (2000).

[58] C. W. Misner, K. S. Thorne, and J. A. Wheeler, Gravitation (Freeman, San Francisco, 1973).

[59] http://www.srl.caltech.edu/ shane/sensitivity/; S.L. Larson, W. A. Hiscock, and R. W. Hellings, Phys. Rev. D 62, 062001 (2000); S. L. Larson, R. W. Hellings, and W. A. Hiscock, Phys. Rev. D 66, 062001 (2002).

[60] K. Glampedakis and D. Kennefick, Phys. Rev. D 66, 044002 (2002).

[61] M.E. Pati and C.M. Will, Phys. Rev. D 62, 124015 (2000).

[62] J. D. Bekenstein, Astrophys. J. 183, 657 (1973).

[63] W. H. Press, Phys. Rev. D 15, 965 (1977). 